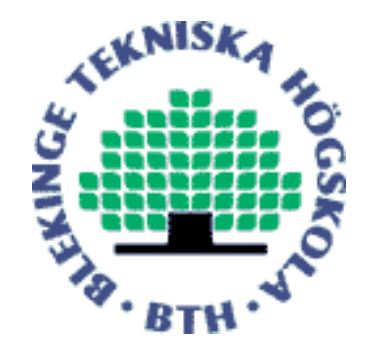

Copyright @ 2010 IEEE.

Citation for the published paper:

Title:

Detection of Moving Targets by Focusing in UWB SAR-Theory and Experimental Results

Author:

Viet Thuy Vu, Thomas Sjögren, Mats Pettersson, Anders Gustavsson, Lars Ulander

Journal:

IEEE Transactions on Geoscience and Remote Sensing

Year:

2010

Vol:

48

Issue:

10

Pagination:

$3799-3815$

URL/DOI to the paper:

This material is posted here with permission of the IEEE. Such permission of the IEEE does not in any way imply IEEE endorsement of any of BTH's products or services Internal or personal use of this material is permitted. However, permission to reprint/republish this material for advertising or promotional purposes or for creating new collective works for resale or redistribution must be obtained from the IEEE by sending a blank email message to pubs-permissions@iee.org.

By choosing to view this document, you agree to all provisions of the copyright laws protecting it. 


\title{
Detection of Moving Targets by Focusing in UWB SAR-Theory and Experimental Results
}

\author{
Viet Thuy Vu, Student Member, IEEE, Thomas K. Sjögren, Student Member, IEEE, \\ Mats I. Pettersson, Member, IEEE, Anders Gustavsson, and Lars M. H. Ulander, Senior Member, IEEE
}

\begin{abstract}
Moving-target detection in ultrawideband (UWB) synthetic aperture radar (SAR) is associated with long integration time and must accommodate azimuth focusing for reliable detection. This paper presents the theory on detection of moving targets by focusing and experimental results on single-channel SAR data aimed at evaluating the detection performance. The results with respect to both simulated and real data show that the ability to detect moving targets increases significantly when applying the proposed detection technique. The improvement in signal-to-clutter noise ratio, which is a basic requisite for evaluating the performance, reaches approximately $20 \mathrm{~dB}$, using only single-channel SAR data. This gain will be preserved for the case of multichannel SAR data. The reference system for this study is the airborne UWB low-frequency SAR Coherent All RAdio BAnd Sensing II.
\end{abstract}

Index Terms-Coherent All RAdio BAnd Sensing (CARABAS)-II, detection, fast backprojection, fast factorized backprojection (FFBP), moving target, multichannel, single channel, synthetic aperture radar (SAR), ultrawideband (UWB), UWB chirp scaling (UCS).

\section{INTRODUCTION}

$\mathbf{O}$ VER the last decades, synthetic aperture radar (SAR) has attracted considerable interest as the number of applications in geoscience, remote sensing, surveillance, and reconnaissance increases. The ability to effectively collect data in severe conditions, such as rain, clouds, and/or darkness, is considered to be the main advantage of SAR systems as compared to other imaging sensors. Ultrawideband (UWB) SAR is understood as SAR systems utilizing either a large absolute bandwidth or a large fractional bandwidth signal and a wide antenna beamwidth. Examples of experimental UWB SAR systems are Coherent All RAdio BAnd Sensing (CARABAS)-II operating in the lower very high frequency (VHF) band from 20 to $90 \mathrm{MHz}$ [1], LORA in the VHF and UHF bands from 200 to $800 \mathrm{MHz}$ [2], P-3 with a bandwidth of $515 \mathrm{MHz}$ in the VHF/UHF bands at 215-900 $\mathrm{MHz}$ [3], ground-based BoomSAR with a spectral response extending from 50 to

Manuscript received January 30, 2009; revised June 26, 2009, November 30, 2009, and March 8, 2010. Date of publication May 27, 2010; date of current version September 24, 2010. This work was supported in part by the Swedish Defence Research Agency, by Saab Bofors Dynamics, by Saab Microwave Systems, and by RUAG Space.

V. T. Vu, T. K. Sjögren, and M. I. Pettersson are with the Blekinge Institute of Technology, 37225 Ronneby, Sweden (e-mail: viet.thuy.vu@bth.se).

A. Gustavsson is with the Swedish Defence Research Agency (FOI), 58111 Linköping, Sweden.

L. M. H. Ulander is with the Swedish Defense Research Agency (FOI), 581 11 Linköping, Sweden, and also with the Chalmers University of Technology, 41296 Gothenburg, Sweden.

Digital Object Identifier 10.1109/TGRS.2010.2048572
$1200 \mathrm{MHz}$ [4], and PAMIR with a very high simultaneous bandwidth of $1820 \mathrm{MHz}$ in X-band [5]. Such systems usually enable high-resolution imaging. Systems operating at low radar frequencies, e.g., CARABAS, allow one to detect changes in dense forested areas or under camouflage [6]. Such applications are of interest to both military and civilian end users.

In this paper, we concentrate on another application of UWB SAR: detection of moving targets, which is most commonly realized by ground moving-target indication (GMTI) implementations based on antenna array solutions. A moving object is displaced and defocused as an elliptic or hyperbolic curve in a SAR image [7]. For conventional SAR, i.e., small bandwidth signal and narrow beamwidth, the displacement and defocusing in a SAR image are mainly caused by movements in the range and azimuth directions, respectively. For UWB SAR, objects moving in the range direction can be both displaced and defocused in a SAR image due to long integration time. The detection of moving targets, however, cannot be based on such features. A stationary target with an elliptic or hyperbolic shape can give rise to a false detection. Disappearance of moving targets in a SAR image is also possible due to the dispersion of the energy reflected from them. In many cases, the target may be fully obscured by surrounding clutter.

Several moving-target detection methods have been proposed. The detection of moving targets can be based on Doppler effect [8]. However, the detection is only possible if the speed of the target is high enough to produce Doppler frequencies which are distinguishable from the surrounding stationary clutter. Alternatively, moving-target detection can be achieved by using displaced phase center antenna (DPCA) [9] or space time adaptive processing (STAP) [10] techniques. Access to an array antenna gives the opportunity to also correct for the displacement caused by the SAR process. An overview of multichannel SAR GMTI is given in [11]. However, such techniques are not available for single-channel SAR data. The detection can also be based on the phenomenon that the shadow projected by the target will produce zeros in a SAR image [12]. Strong backscattering from the background is required for this approach. Detection based on phase errors caused by moving targets is suggested in [13]. A complex SAR image is divided into patches, where the phase error is estimated separately in each patch. The magnitude of the phase-error estimate is measured and, if it exceeds a given threshold, indicates the presence of a moving target. In addition, there are also new detection methods based on, for example, the capabilities of dual-frequency millimeter-wave SAR with monopulse processing for GMTI [14] or PolSAR imagery for movingtarget detection/ship detection [15]. 
The goal of this paper is to present the theory of the movingtarget detection by focusing technique in UWB SAR and experimental results aimed at evaluating the gain in detection ability. This detection technique is based on the detection scheme proposed in [16] and the optimum relative speed discretization derived in [17]. The experimental results upon single-channel UWB low-frequency SAR data show a significant improvement in detection ability achieved with this detection technique. This improvement will be preserved when the detection technique is performed on multichannel SAR data. The moving targets in our experiments are selected in the areas with low backscattering. This makes the evaluation of the gain in detection ability more efficient. In the experiments, we use both real and simulated data based on the airborne UWB low-frequency SAR CARABAS-II. The real data have been collected by CARABAS-II in two different field campaigns.

This paper is organized as follows. Section II briefly describes the likelihood ratio test (LRT) for moving-target detection. Section III describes in detail the relative speed concept. Issues related to moving-target focusing are presented in Section IV. Section V presents the theory of the movingtarget detection by focusing technique and experiments upon simulated data. Experimental results on real data collected in the two field campaigns are given in Sections VI and VII. Section VIII provides the conclusions and outlines future work.

\section{LRT FOR MOVING-TARGET DETECTION}

The most commonly used radars for GMTI are based on antenna array solutions but often without SAR capabilities. However, SAR systems are becoming more and more important for GMTI since, in SAR GMTI systems, the moving target will not only be detected but also imaged in its surroundings.

For moving-target detection, two hypotheses on the sampled radar echo $\mathbf{Y}$ can be given: A moving object exists, i.e., the received signal consists of the reflection from the moving object $\mathbf{S}$, (hypothesis $H_{1}$ ), or no moving object exists (hypothesis $H_{0}$ ). In both cases, the received signal contains clutter connected to the radar backscattering $\mathbf{C}$ and noise $\mathbf{N}$ originating from thermal noise in the system

$$
\begin{aligned}
& H_{1}: \mathbf{Y}=\mathbf{S}+\mathbf{C}+\mathbf{N} \\
& H_{0}: \mathbf{Y}=\mathbf{C}+\mathbf{N}
\end{aligned}
$$

The LRT for moving-target detection, known as an optimal detection scheme in the maximum likelihood sense, is given by

$$
\Lambda=\frac{P\left(\mathbf{Y} \mid H_{1}\right)}{P\left(\mathbf{Y} \mid H_{0}\right)} .
$$

Different optimal schemes for detection in the maximum likelihood sense have been proposed. In [18], with an assumption of Gaussian probability density functions for clutter and noise, the optimal scheme for detection can be written as

$$
\Lambda=\max \left|\mathbf{Y}^{\mathrm{T}} \mathbf{R}^{-1} \mathbf{S}^{*}\left(f_{D}, k_{D}\right)\right| \begin{cases}\geq \lambda, & \text { decision for } H_{1} \\ <\lambda, & \text { decision for } H_{0}\end{cases}
$$

where $\mathbf{R}$ is the covariance matrix and $\lambda$ is a suitable threshold. This is equivalent to multiplying the received signal by a weighting vector

$$
\mathbf{w}_{\mathrm{opt}}=\mathbf{R}^{-1} \mathbf{S}^{*}\left(f_{D}, k_{D}\right)
$$

where knowledge of Doppler frequency $f_{D}$ and Doppler chirp rate $k_{D}$ can be obtained by an open- or closed-loop technique [18].

Another solution for LRT in the case of local backprojection (LBP) [19] SAR processing is given in [16] as

$$
\begin{aligned}
\Lambda=\mid \sum_{l=1}^{N_{a}} \frac{1}{R_{c, l}^{2}} \sum_{m=0}^{N_{f}-1} e^{j k_{m} r_{l}} \mathbf{A}_{m l}^{H}\left(\theta_{l}^{\prime}\right) & \\
& \times\left.\mathbf{R}_{m l}^{-1} \tilde{\mathbf{Y}}_{m l}\right|^{2} \begin{cases}\geq \lambda, & \text { decision for } H_{1} \\
<\lambda, & \text { decision for } H_{0}\end{cases}
\end{aligned}
$$

where $l$ is the subaperture number, $m$ is the frequency number, $r_{l}$ is the range to a moving target in the $l$ th subaperture beam, $k_{m}$ is the wavenumber at the $m$ th frequency, $R_{c, l}$ is the range from the center of the $l$ th subaperture to subimage, $\tilde{\mathbf{Y}}_{m l}$ is the subaperture beam measurement vector, $\mathbf{R}_{m l}$ is the covariance matrix, and $\mathbf{A}_{m l}\left(\theta_{l}^{\prime}\right)$ is the steering vector at $m$ and $l$ for the moving target. If only single-channel data are used, the steering vector $\mathbf{A}_{m l}\left(\theta_{l}^{\prime}\right)$ is equal to one. The detection here is carried out as follows: First, multichannel subapertures are formed from multichannel SAR data. The clutter suppression is then performed on these subapertures by the covariance $\mathbf{R}_{m l}$ and the target steering vector $\mathbf{A}_{m l}\left(\theta_{l}^{\prime}\right)$. The subapertures are combined together using different $r_{l}$ 's (dependent on the difference velocity of the platform velocity and the target velocity) to focus the target energy. In [16], the clutter suppression step was tested with good results on the multichannel C-band data. In this paper, the experiments on the single-channel UWB lowfrequency SAR data shows a significant increase in detection ability when using the correct difference velocity in the SAR image formation.

\section{NRS}

Let the ground coordinates be given by a Cartesian coordinate system $(\xi, \eta, \zeta)$, as shown in Fig. 1. If all movements are assumed to have constant speeds, i.e., no acceleration, and to be linear, the movement of the target can then be represented by the coordinates

$$
\begin{aligned}
& \xi_{\mathrm{tg}}(t)=v_{\xi} \cdot\left(t-t_{0}\right)+\xi_{0} \\
& \eta_{\mathrm{tg}}(t)=v_{\eta} \cdot\left(t-t_{0}\right)+\eta_{0} \\
& \zeta_{\mathrm{tg}}(t)=0
\end{aligned}
$$

where $t_{0}$ is the time at the minimum range $r_{0}$. The ground coordinates of the target at $r_{0}$ are $\xi_{0}$ and $\eta_{0}$. On the other hand, the linear movement of the platform is given by

$$
\begin{aligned}
\xi_{\mathrm{pl}}(t) & =v_{\mathrm{pl}} t \\
\eta_{\mathrm{pl}}(t) & =0 \\
\zeta_{\mathrm{pl}}(t) & =h .
\end{aligned}
$$




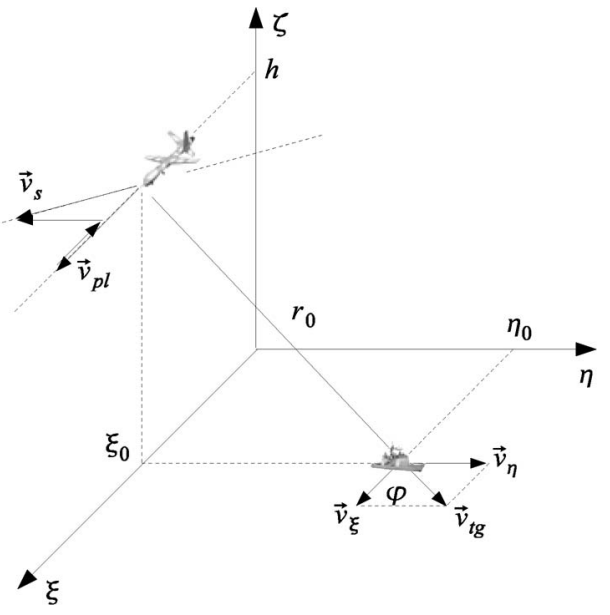

Fig. 1. SAR system geometry. $\vec{v}_{\mathrm{pl}}$ is the velocity, and $h$ is the altitude of the platform. The velocity of the target $\vec{v}_{\text {tg }}$ can be analyzed by velocities $\vec{v}_{\xi}$ and $\vec{v}_{\eta}$ in $\xi$ and $\eta$ directions, respectively, and $\varphi$ is the moving direction of the target with respect to the flight track. The ground coordinates $\xi_{0}$ and $\eta_{0}$ are positions at the minimum range $r_{0}$, and $\vec{v}_{s}$ is the difference vector of $\vec{v}_{\mathrm{pl}}$ and $\vec{v}_{\mathrm{tg}}$.

Given the coordinates of the ground target and the platform, the slow-time-dependent range is defined by

$$
\bar{r}_{t}(t)=\sqrt{\left[\xi_{\mathrm{pl}}(t)-\xi_{\mathrm{tg}}(t)\right]^{2}+\eta_{\mathrm{tg}}^{2}(t)+\zeta_{\mathrm{pl}}^{2}(t)} .
$$

A moving target can be considered as a stationary target in the SAR formation process if the platform carrying the SAR equipment is assumed to move with the difference velocity $\vec{v}_{s}$ of the original platform velocity $\vec{v}_{\mathrm{pl}}$ and the target velocity $\vec{v}_{\mathrm{tg}}[16]$. Hence, the slow-time-dependent range in image coordinates $(x, r)$ can also be written as

$$
\bar{r}_{t}(t)=\sqrt{\left(v_{s} t-x_{0}\right)^{2}+r_{0}^{2}}
$$

where $x_{0}$ is the platform position at the minimum range The terms $r_{0}, v_{s}, t_{0}$, and $x_{0}$ are connected together by the relationship

$$
x_{0}=v_{s} t_{0} .
$$

If we substitute (6) and (7) into (8) and then equalize the coefficients of $t^{2}, t$, and the constants in (8) and (9), $v_{s}$ and the position of the moving target in the image coordinates are found to be

$$
\begin{aligned}
v_{s} & =\sqrt{\left(v_{\mathrm{pl}}-v_{\xi}\right)^{2}+v_{\eta}^{2}} \\
r_{0} & =\sqrt{\eta_{0}^{2} \cdot\left[1+\left(\frac{v_{\eta}}{v_{\mathrm{pl}}-v_{\xi}}\right)^{2}\right]+h^{2}} \\
x_{0} & =\frac{v_{s}}{v_{\mathrm{pl}}}\left(\xi_{0}-\frac{v_{\eta}}{v_{\mathrm{pl}}-v_{\xi}} \eta_{0}\right) .
\end{aligned}
$$

The normalized relative speed (NRS) $\gamma_{t}$ is defined by the length of the difference velocity $\vec{v}_{s}$, which is normalized with respect to the platform speed $v_{\mathrm{pl}}$

$$
\gamma_{t}=\sqrt{\frac{\left(v_{\mathrm{pl}}-v_{\xi}\right)^{2}+v_{\eta}^{2}}{v_{\mathrm{pl}}^{2}}} .
$$

With such, (9) can be rewritten in a more convenient form as

$$
\bar{r}_{t}(t)=\sqrt{\gamma_{t}^{2} \cdot\left(v_{\mathrm{pl}} t-x_{0}^{\prime}\right)^{2}+r_{0}^{2}}
$$

where

$$
x_{0}^{\prime}=\xi_{0}-\frac{v_{\eta}}{v_{\mathrm{pl}}-v_{\xi}} \eta_{0} .
$$

Equation (15) is called the focusing equation and can be found also in [16]. A moving target can be focused in the SAR image simply by scaling the speed of the platform with $\gamma_{t}$.

\section{Moving-Target Focusing}

The focusing approach can be carried out differently, depending on which image formation algorithm is used. In general, algorithms can be divided into two groups: time- and frequency-domain algorithms. Both of them have been proved to be applicable to the focusing approach [20], [21].

Global backprojection (GBP) [22], which is a time-domain algorithm, has been used in [20] to focus moving target for detection. The moving-target focusing is obtained exactly by (9). However, this algorithm normally requires extremely long processing time. To save the processing time, we can use faster time-domain algorithms such as LBP [19] or fast backprojection [23]. To avoid distance compensation and subimage shifts associated with LBP [24], we propose to use PBP. Aside from this, fast factorized backprojection (FFBP) [25], [26] can also be an option for the moving-target focusing.

In [21], the moving-target focusing is based on a frequencydomain algorithm called range migration (RM) [27]. A new wavenumber in azimuth $k_{x}$ scaled with $\gamma_{t}$ to focus the moving target is given by

$$
k_{x}^{\prime}=\frac{2 \pi f_{x}}{\gamma_{t} v_{\mathrm{pl}}}
$$

where $f_{x}$ is the azimuth frequency. However, RM requires the Stolt interpolation, and this leads to high computational complexity. UWB chirp scaling (CS) (UCS) [28], which is a candidate for UWB SAR data processing, does not require interpolation and can be efficiently performed by complex multiplications and fast Fourier transforms; it thus has the advantage of short processing time and is also selected as a candidate for the focusing approach. In this section, we present guidelines to focus moving targets using PBP and UCS.

\section{A. Moving-Target Focusing Using PBP}

In PBP [23], two concepts-polar and slant-range images-are used. Similar to LBP, PBP divides the complete aperture into subapertures. The selection of subaperture size is based on the SAR system parameters and has a direct influence on image quality and processing time. However, any selection must ensure that the phase error is smaller than $\pi / 8$ (far-field condition). From the center of each subaperture, a polar grid $(\cos \hat{\alpha}, \hat{r})$ is created. The polar grid must cover the full 
slant-range image and meet the Nyquist sampling requirements for $\hat{\alpha}$ and $\hat{r}$

$$
\begin{aligned}
\Delta \cos \hat{\alpha} & <\frac{\mathrm{c}}{2 f_{\max } L_{s}} \\
\Delta \hat{r} & <\frac{\mathrm{c}}{2 B}
\end{aligned}
$$

where $f_{\max }$ is the maximum radar signal frequency, $L_{s}$ is the subaperture size, $\mathrm{c}$ is the speed of light, and $B$ is the signal bandwidth. In PBP, the polar images are retrieved by superposition of backprojected data in polar grids. The range from a position in the $l$ th subaperture to a pixel $(m, n)$ of the $l$ th polar image is determined by

$$
\tilde{r}_{l, m, n}(t)=\sqrt{\left(v_{\mathrm{pl}} t-x_{l}-\hat{r}_{n} \cos \hat{\alpha}_{m}\right)^{2}+\left(\hat{r}_{n} \sin \hat{\alpha}_{m}\right)^{2}} \text {. }
$$

The slant-range image is obtained by linear mapping from all the polar images. The range from the center of the $l$ th subaperture to a slant-range image pixel $\left(x_{i}, r_{j}\right)$ and the cosine of the angle between the range and the flight track vectors are derived as

$$
\begin{aligned}
\bar{r}_{l, i, j} & =\sqrt{\left(x_{l}-x_{i}\right)^{2}+r_{j}^{2}} \\
\cos \left(\bar{\alpha}_{l, i, j}\right) & =\frac{x_{l}-x_{i}}{\bar{r}_{l, i, j}} .
\end{aligned}
$$

Moving-target focusing can be obtained by scaling the speed of the platform with NRS in (20), i.e., focusing in the polar image formation

$$
\tilde{r}_{l, m, n}(t)=\sqrt{\gamma_{t}^{2} \cdot\left(v_{\mathrm{pl}} t-x_{l}-\hat{r}_{n} \cos \hat{\alpha}_{m}\right)^{2}+\left(\hat{r}_{n} \sin \hat{\alpha}_{m}\right)^{2}} .
$$

The focusing can also be carried out by scaling the speed of the platform with $\gamma_{t}$ in (21), i.e., focusing in the slant-range image formation

$$
\bar{r}_{l, i, j}=\sqrt{\gamma_{t}^{2} \cdot\left(x_{l}-x_{i}\right)^{2}+r_{j}^{2}} .
$$

For FFBP, the focusing can be performed on either any beam forming stage or the image formation stage. This issue is presented in detail in [29] and [30].

\section{B. Moving-Target Focusing Using UCS}

Due to the approximations in CS [31], it may only be valid for a limited number of SAR systems which utilize small fractional bandwidth and narrow beamwidth. A proposal to apply nonlinear CS (NCS) to process CARABAS-II data has been given in [32]. However, with such proposal, we must reduce the pulse repetition frequency (PRF) by considering one out of several adjacent radar echo data to keep the term $\beta$ real at all azimuth frequencies with a high possibility of aliasing

$$
\beta=\sqrt{1-\left(\frac{\mathrm{c} f_{x}}{2 f_{c} v_{\mathrm{pl}}}\right)^{2}}
$$

where $f_{c}$ is the center frequency and $f_{x}$ is the azimuth frequency. The idea behind UCS is to manipulate a higher speed
TABLE I

PARAmeters Used in The MEASUREMENT CAMPAign IN Simrishamn

\begin{tabular}{cc}
\hline \hline Parameter & Value \\
\hline The highest frequency processed & $82 \mathrm{MHz}$ \\
The lowest frequency processed & $22 \mathrm{MHz}$ \\
Platform speed $v_{p l}$ & $128 \mathrm{~m} / \mathrm{s}$ \\
Full aperture length & $19200 \mathrm{~m}$ \\
Full aperture positions & 20480 \\
Flight altitude & $3700 \mathrm{~m}$ \\
Minimum range to the aim point & $7150 \mathrm{~m}$ \\
PRF & $137 \mathrm{~Hz}$ \\
\hline \hline
\end{tabular}

of the SAR platform in processing, i.e., scaling the speed of the SAR platform with NRS $\gamma_{u}>1$, to keep $\beta$ in real at all azimuth frequencies [28]

$$
\beta=\sqrt{1-\left(\frac{c f_{x}}{2 f_{c} \gamma_{u} v_{p l}}\right)^{2}} .
$$

The condition for $\gamma_{u}$ is retrieved directly from (26) as

$$
\gamma_{u}>\frac{\lambda_{c}}{4 \Delta L}
$$

where $\Delta L$ is the space between two adjacent SAR platform positions. Defocusing caused by $\gamma_{u}$ will be compensated in the phase functions. In addition, the space-variant RM existing in UWB SAR is handled by using the nonlinear FM filtering method [33], i.e., NCS. An extra phase filter $\psi_{0}\left(f_{t}, f_{\tau}\right)$ in the 2-D frequency domain is introduced in [28] as

$$
\psi_{0}\left(f_{t}, f_{\tau}\right)=\exp \left[i \pi Y\left(f_{t}\right) f_{\tau}^{3}+i \pi Z\left(f_{t}\right) f_{\tau}^{4}\right]
$$

where $f_{\tau}$ is the range frequency, $Y\left(f_{t}\right)$ and $Z\left(f_{t}\right)$ are the azimuth frequency-varying phase filter coefficients. The UCS phase functions of CS, range compression, and residual phase correction, which include the compensation for the defocusing caused by $\gamma_{u}$, are presented in details in [28]. For UCS, movingtarget focusing can be reached by scaling again the platform speed using the true NRS $\gamma_{t}$ in (26) as

$$
\beta=\sqrt{1-\frac{1}{\gamma_{t}^{2}}\left(\frac{\mathrm{c} f_{x}}{2 f_{c} \gamma_{u} v_{\mathrm{pl}}}\right)^{2}} .
$$

\section{Moving-Target Focusing Tests}

In moving-target focusing tests, we use simulated data based on the parameters of the airborne UWB low-frequency SAR CARABAS-II [1] in order to keep consistency with the later experiments. These parameters were used in the data collection campaign in the Simrishamn area in 1999, and they are given in Table I. Experiments on real data will be given in Sections VI and VII.

To test the ability of focusing a moving target with NRS, we assume that there are two known moving point targets. One moves with speed $v_{\mathrm{tg}}=5.34 \mathrm{~m} / \mathrm{s}$ toward $\varphi=81.5^{\circ}$ with respect to the flight track, as shown in Fig. 1 and the other toward $\varphi=-65.2^{\circ}$ with the same speed. Estimated NRSs are 
(a)

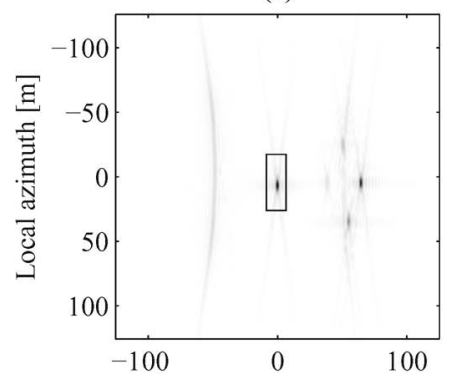

(b)

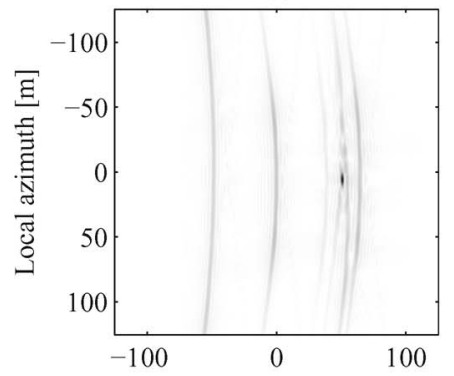

(c)

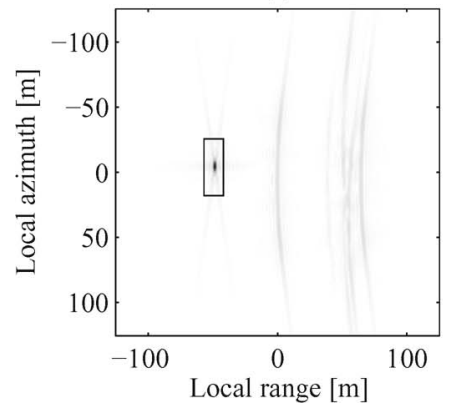

(d)

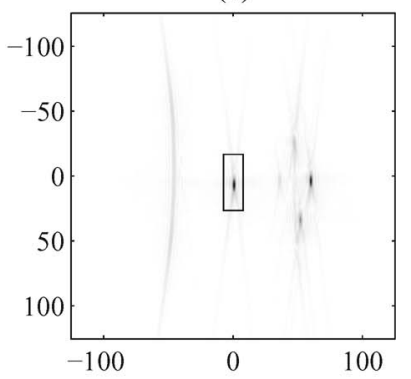

(e)

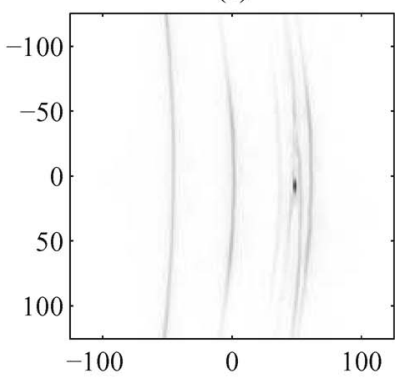

(f)

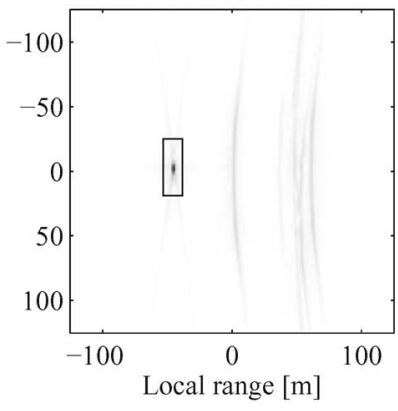

Fig. 2. SAR images of the simulated stationary and moving targets processed by PBP and UCS. The integration angle $\theta=15^{\circ}$ is chosen arbitrarily. In the SAR scene, the moving target with $\gamma_{t}=1.0378$, which appears on the lefthand side of the SAR images, has an RCS of $\sigma=1$ and the one with $\gamma_{t}=$ 0.959 on the right-hand side $\sigma=0.25$. There are one stationary target with $\sigma=1$ in the middle of SAR images and four other stationary targets on the right-hand side with $\sigma=0.25,0.5,0.75$, and 1 surrounding the moving target $\gamma_{t}=0.959$. The black rectangles mark the areas of detection (containing the moving target $\gamma_{t}=1.0378$ and $\sigma=1$ ) and the areas of reference (containing the stationary target and $\sigma=1$ ), which are used for evaluation purposes. Other targets are used mainly for moving-target detection illustration. (a) Original SAR image processed by $\mathrm{PBP}\left(\gamma_{t}=1\right)$. (b) SAR image processed by PBP with $\gamma_{t}=0.959$. (c) SAR image processed by PBP with $\gamma_{t}=1.0378$. (d) Part of the original SAR image processed by UCS $\left(\gamma_{t}=1\right)$ ). (e) Part of SAR image processed by UCS with $\gamma_{t}=0.959$. (f) Part of SAR image processed by UCS with $\gamma_{t}=1.0378$.

$\gamma_{t}=0.959$ and $\gamma_{t}=1.0378$. The radar cross section (RCS) of the target with $\gamma_{t}=1.0378$ is normalized to $\sigma=1$, the other with $\gamma_{t}=0.959$ and $\sigma=0.25$. In addition, a number of stationary point targets with RCS $\sigma=0.25,0.5,0.75$, and 1 are also present in order to investigate the effects caused by the focusing approach to a SAR image. The thermal noise is assumed to be additive white Gaussian noise at a level of $-10 \mathrm{~dB}$. Fig. 2(a) shows the $250 \mathrm{~m} \times 250 \mathrm{~m}$ SAR image of the five stationary targets and two moving targets processed by PBP. However, the size of a SAR image processed by UCS is specified by the considered number of aperture positions and the number of range data. Fig. 2(d) shows part of the original SAR image processed by UCS.

The moving targets are displaced and defocused in the SAR images. One appears on the left-hand side of the SAR images as elliptic curves, and the other on the right-hand side is surrounded by four stationary targets. Fig. 2(b) and (e) shows the SAR images of the stationary point target and the moving targets after applying the focusing approach using a known NRS $\gamma_{t}=0.959$. The stationary targets in the center and surrounding the moving target on the right-hand side of the SAR images are now defocused and appear as elliptic curves. The moving target on the left-hand side of the SAR images is strongly defocused and appears almost as a straight line. Meanwhile, the moving target surrounded by the stationary targets on the righthand side of the SAR images is now focused to the original shape (point) and can be seen clearly. Similar effects can be seen in the SAR images in Fig. 2(c) and (f) after applying the focusing approach using another known NRS $\gamma_{t}=1.0378$. The superposition of the hyperbolic curves caused by the defocusing of the stationary targets, as well as the moving target, can result in higher energy concentration in some image pixels.

\section{Detection of Moving TARgets By Focusing}

As illustrated, a moving object can be focused to its original shape in the SAR image by simply processing SAR data with NRS, i.e.,scaling the platform speed with NRS, while any stationary objects are instead defocused. However, this focusing approach can only be carried out if motion parameters of the moving object, e.g., speed and motion direction, are known. In reality, these parameters are not usually known and therefore require complex estimation procedures if they are to be retrieved. These procedures can be applied if, and only if, the presence of a moving object is indicated; in other words, the moving object must be detected first before applying estimation procedures.

For detection, different blind hypotheses $\gamma_{p}$, the so-called NRS under test, must be tested on either (23) or (24) and (29) until the optimum moving-target focusing for detection is reached. In this paper, the subscript $p$ is used to denote the processed variables. The energy reflected from moving objects, as well as stationary objects, at each hypothesis $\gamma_{p}$ is therefore either concentrated or dispersed. If there is a moving object with $\gamma_{t}$, at the hypothesis $\gamma_{p} \approx \gamma_{t}$, the energy reflected from the moving object is highly concentrated, and the energy reflected from the stationary objects is dispersed due to smearing.

The subsequent problem is then how to adapt the value of $\gamma_{p}$ between the tests. In other words, how large should the step size be between different blind hypotheses in the detection scheme? A tradeoff between the probability of detection and the computational cost must be taken into account. The highest probability of detection can be obtained for the smallest step size. However, depending on the false alarm rate, different step sizes may be used.

\section{A. Optimum Step Size of NRS for Detection}

An optimum quantization step size $\Delta \gamma$ for detection is proposed in [17]. The derivation of the step size is based on the decrease of the SAR image intensity of a point target given 


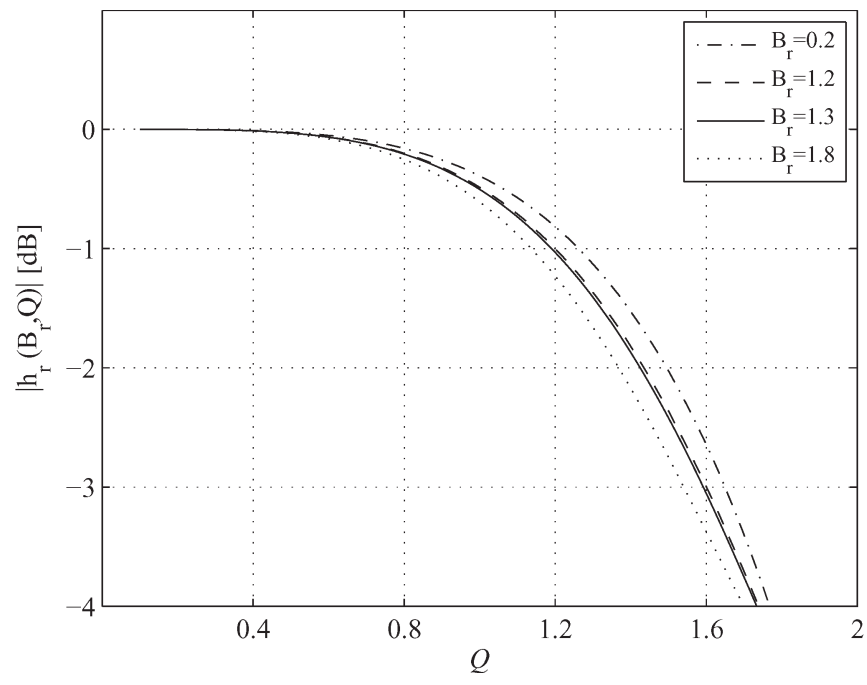

Fig. 3. Absolute values of $\left|h_{r}\left(\gamma_{p}, t_{0}\right)\right|$ as a function of the fractional bandwidth $B_{r}$ (PAMIR: $B_{r} \approx 0.2$, LORA and P-3: $B_{r} \approx 1.2$, CARABAS-II: $B_{r} \approx 1.3$, and BoomSAR: $\left.B_{r} \approx 1.8\right)$ and $Q$.

by the GBP integral [22]. This decrease can be estimated by the ratio

$$
h_{r}\left(\gamma_{p}, t_{0}\right)=\frac{\int_{-\infty}^{+\infty} g\left(t, \sqrt{\gamma_{p}^{2} \cdot\left[v_{\mathrm{pl}} t-x_{p}\left(t_{0}\right)\right]^{2}+r_{p}^{2}\left(t_{0}\right)}\right) d t}{\int_{-\infty}^{+\infty} g\left(t, \sqrt{\gamma_{t}^{2} \cdot\left[v_{\mathrm{pl}} t-x_{0}\right]^{2}+r_{0}^{2}}\right) d t}
$$

where $g(t, r)$ is the band-limited radar pulse after range compression. Hence, the denominator of the ratio (30) defines the peak intensity of a point target, i.e., $\gamma_{p}=\gamma_{t}$. The numerator determines the intensity of a point target corresponding to a blind hypothesis $\gamma_{p}$. An incorrect hypothesis smears the point target into the shape of an ellipse or a hyperbola and thereby causes a reduction of the ratio (30).

If we assume that a chirp signal is transmitted, Fig. 3 shows the absolute values of the integral (30) as a function of the fractional bandwidth $B_{r}$ and the scalar variable $Q$

$$
Q=v_{\mathrm{pl}} t_{i} \sqrt{\frac{\pi f_{c}\left|\gamma_{p}^{2}-\gamma_{t}^{2}\right|}{2 \mathrm{c} r_{0}}}
$$

where $t_{i}$ is the integration time. With the parameters given in Table I, the CARABAS-II fractional bandwidth is estimated about 1.3. Assuming that the maximum acceptable ratio $\left|h_{r}\left(\gamma_{p}, t_{0}\right)\right|$ is given by $h_{\mathrm{lim}}$, then the condition for $\Delta \gamma$ can then be found using the following inequality:

$$
\left|h_{r}\left(\gamma_{t} \pm \frac{\Delta \gamma}{2}, t_{0}\right)\right| \leq h_{\lim }
$$

A threshold $h_{\text {lim }}$ should be set as desired to meet the demand of the acceptable detection failure. With the threshold $h_{\text {lim }}$, the optimum discretization step can be approximated by

$$
\Delta \gamma \approx \frac{Q^{2} c r_{0}}{2 \pi f_{c} \gamma_{t} v_{\mathrm{pl}}^{2} t_{i}^{2}}
$$

(a)

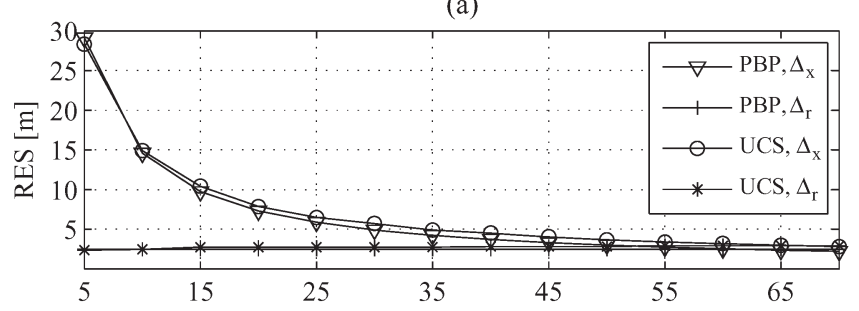

(b)

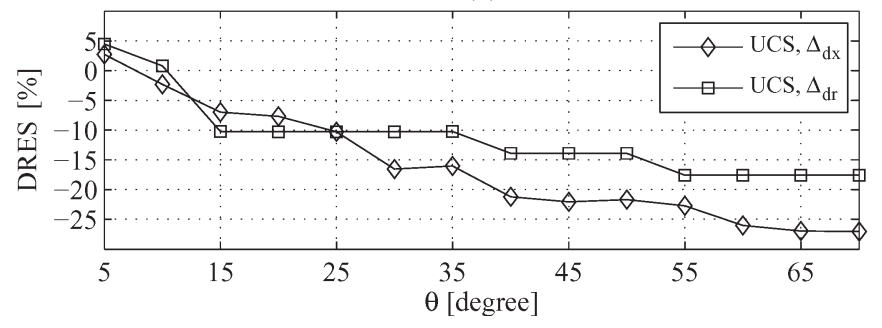

Fig. 4. (a) Spatial resolutions in azimuth $\Delta_{x}$ and range $\Delta_{x}$ (RES) obtained by PBP and UCS at different integration angles. (b) UCS differential resolution (DRES) with reference to PBP.

Equation (33) is derived for short apertures. However, it is shown in [17] that this approximate solution can also be applied to long apertures with a discretization step error of less than $10 \%$, i.e., the discretization step derived with the approximation of short apertures (33) is always smaller than the discretization step for long apertures. The range of NRS being tested can be derived from another form of (14) as

$$
\gamma_{t}=\sqrt{\left(\frac{v_{\mathrm{tg}}}{v_{\mathrm{pl}}}\right)^{2}-2\left(\frac{v_{\mathrm{tg}}}{v_{\mathrm{pl}}}\right) \cos \varphi+1}
$$

where $\varphi$ is the moving direction of the object. The minimum and maximum values of $\gamma_{t}$ correspond to moving directions of $0^{\circ}$ and $180^{\circ}$, respectively

$$
\begin{gathered}
\gamma_{t, \min }=1-\max \left\{\frac{v_{\mathrm{tg}}}{v_{\mathrm{pl}}}\right\} \\
\gamma_{t, \max }=1+\max \left\{\frac{v_{\mathrm{tg}}}{v_{\mathrm{pl}}}\right\} .
\end{gathered}
$$

\section{B. Procedures and Practical Considerations for Detection}

The procedure to perform the moving-target detection by focusing technique in this paper can be listed as follows:

1) processing data with different hypotheses in SAR image formation [using either (23) or (24) and (29)];

2) examining image pixels after the data processing;

3) detecting the presence of moving objects based on intensity.

Some practical issues should also be considered in the tests. For example, it may not be necessary and efficient to use the complete aperture for detection. The assumption of no acceleration in Section III is also more likely to hold with small integration times associated with small integration angles. Image pixel spacing is another practice issue since it affects the number of the tested hypotheses and, therefore, the processing time. We propose to use the spatial resolutions, i.e., $-3-\mathrm{dB}$ 


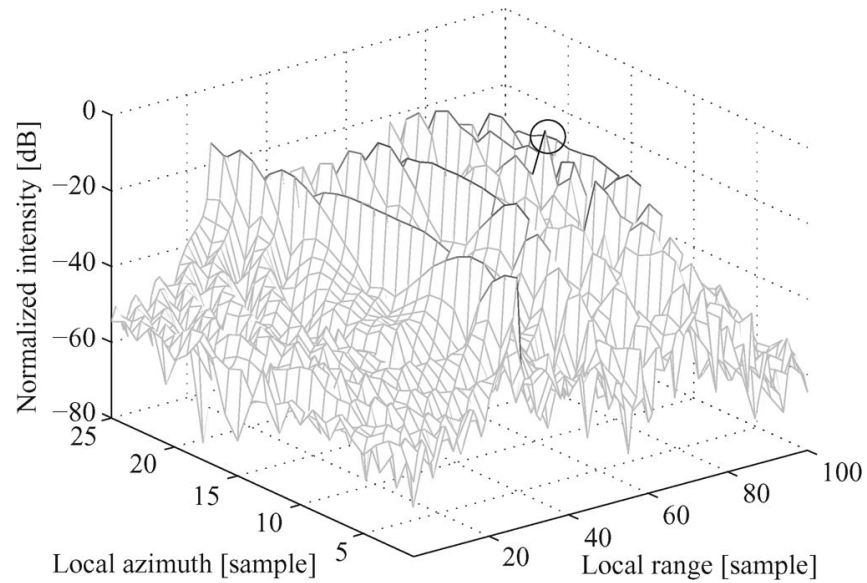

Fig. 5. Measured normalized intensities on 2500 image pixels $10 \mathrm{~m} \times 2.5 \mathrm{~m}$, belonging to the $250 \mathrm{~m} \times 250 \mathrm{~m}$ tested area processed by PBP at the hypothesis $\gamma_{p}=0.96$. The black circle denotes the peak value of normalized intensities at this hypothesis. The peak corresponds to the moving target with $\sigma=0.25$.

widths, in azimuth and range for the image pixel spacing in the detection stage. Fig. 4(a) shows the measurement results on spatial resolutions (RES) obtained by PBP and UCS at different integration angles using the simulated data. The azimuth and range resolutions can also be approximately estimated by [34]

$$
\begin{aligned}
& \Delta_{x} \approx \frac{\mathrm{c}}{2 f_{c} \theta} \\
& \Delta_{r} \approx \frac{\mathrm{c}}{2 B}
\end{aligned}
$$

respectively, where $\theta$ is the processed integration angle and $B$ is the signal bandwidth.

\section{Moving-Target Detection Tests on the Simulated Data}

In this paper, SAR images are represented in local azimuth and local range coordinates. The formats (azimuth 1: azimuth 2, range 1: range 2), (azimuth, range), and azimuth $\times$ range are used to locate an area, a position, and the dimension of an area, respectively.

Moving-target detection tests in this section use the same simulated SAR data in Section IV. The tests follow the detection procedures given in Section V-B. An arbitrary integration angle, e.g., $\theta=15^{\circ}$, is selected for the tests. The corresponding image pixel spacing, which can be seen in Fig. 4(a), should be $10 \mathrm{~m} \times 2.5 \mathrm{~m}$. Different blind hypotheses $\gamma_{p}$ are tested on the whole imaged SAR scene in Fig. 2(a), i.e., $250 \mathrm{~m} \times$ $250 \mathrm{~m}$. Depending on the expected detection ability, different optimum discretization step sizes can be found based on (33). In general, we can decide on an optimum quantization step size corresponding to the threshold $h_{\mathrm{lim}}=-3 \mathrm{~dB}$, i.e., giving a maximum loss of $3 \mathrm{~dB}$ from the peak intensity. With such threshold, (33) results in the optimum discretization step size $\Delta \gamma \approx 0.005$. The range of hypotheses $\gamma_{p}$ can be estimated by (35) and (36). It is assumed that a slow moving point target is the subject to be detected. The maximum speed of the simulated target is $12.8 \mathrm{~m} / \mathrm{s}$, and the moving direction is not known. Equations (35) and (36) result in a range of different blind hypotheses $\gamma_{p} \in[0.9,1.1]$.

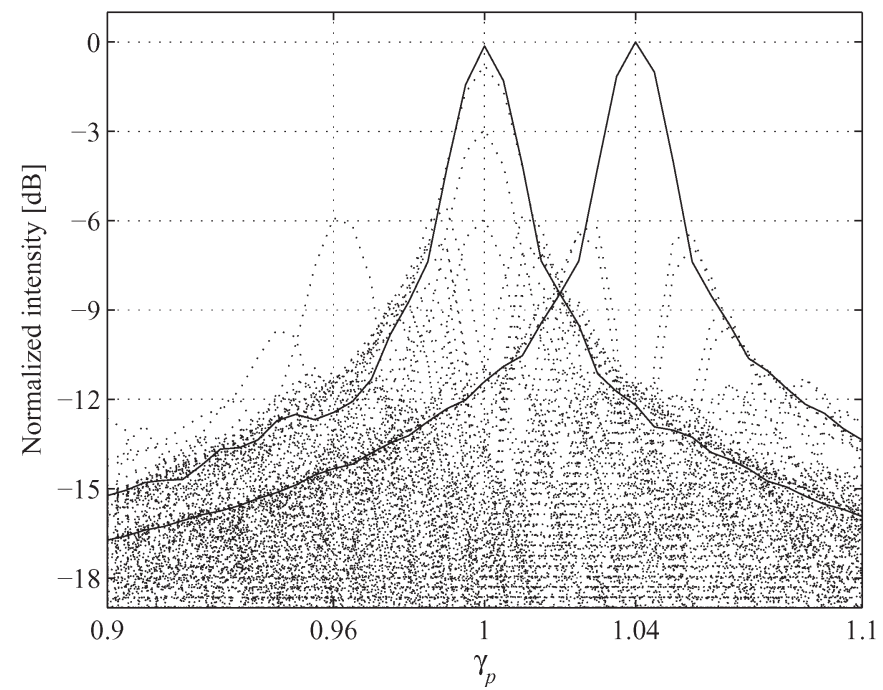

Fig. 6. Measured normalized intensities on 2500 image pixels $10 \mathrm{~m} \times 2.5 \mathrm{~m}$, belonging to the $250 \mathrm{~m} \times 250 \mathrm{~m}$ tested area processed by PBP with different $\gamma_{p}$ 's. The solid black plots denote the peaks of the targets with $\sigma=1$; the peak $-6 \mathrm{~dB}$ at $\gamma_{p}=0.96$ corresponds to the moving target with $\sigma=0.25$.

In the first test in this section, the SAR data are processed by PBP with an image pixel spacing of $10 \mathrm{~m} \times 2.5 \mathrm{~m}$. Fig. 5 shows the measured normalized intensities of the image pixels belonging to the tested area at the hypothesis $\gamma_{p}=0.96$. The number of image pixels within this area is $N_{\text {pixel }}=2500$. The behavior of the normalized intensities belonging to the tested area at all hypotheses $\gamma_{p}$ can be investigated by mapping them in a 2-D plot. Fig. 6 shows the relationship between the NRS being tested and the normalized intensities. The peak values of the normalized intensities are retrieved at the hypotheses $\gamma_{p}=0.96$ and $\gamma_{p}=1.04$, indicating the highest possibility of the presence of the two simulated moving targets. Another is retrieved at the hypothesis $\gamma_{p}=1$, indicating the presence of the simulated stationary target. The detection of the moving target with $N R S \approx 0.96$ indicates that the moving-target detection by focusing technique facilitates detecting weak moving targets which are obscured by strong surrounding stationary targets.

For UCS, we must perform blind tests $\gamma_{p}$ on an area whose dimensions are defined by the number of aperture positions and the number of range data. The data belonging to the areas of interest are then extracted. The image pixel spacing is fixed and depends on the distance between two adjacent aperture positions, i.e., the ratio of the platform speed $v_{\mathrm{pl}}$ to PRF, and the range sampling frequency. With the parameters given in Table I, the image pixel spacing in azimuth is estimated to be $0.9375 \mathrm{~m}$. The range sampling frequency $160 \mathrm{MHz}$ results in the image pixel in a range of $0.9375 \mathrm{~m}$. For the same $250 \mathrm{~m} \times$ $250 \mathrm{~m}$ tested area, which is a part of the SAR image processed by UCS, the number of image pixels rises to $N_{\text {pixel }}=71289$. Fig. 7 shows the plot of the measured normalized intensities in the tested area with respect to NRS under test.

The plot shows that there seems to be more than one moving target with NRS $\approx 0.96$ and more than one moving target with $\mathrm{NRS} \approx 1.04$. However, all normalized intensities around the $\mathrm{NRS} \approx 0.96$ or NRS $\approx 1.04$ belong to adjacent image pixels and are smeared from a single moving target. Such effect is 


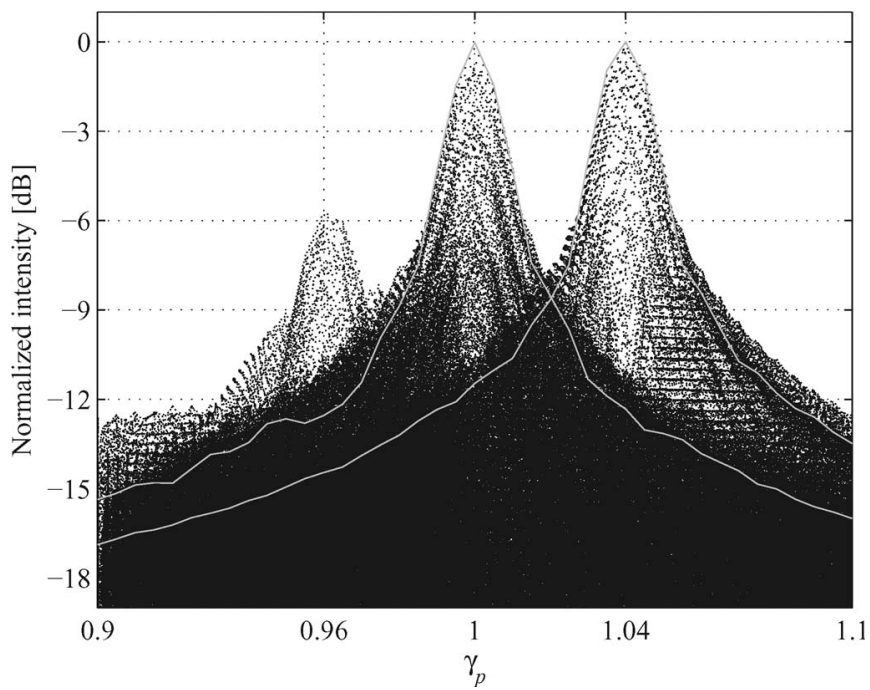

Fig. 7. Measured normalized intensities on 71289 image pixels $0.9375 \mathrm{~m} \times$ $0.9375 \mathrm{~m}$, belonging to the $250 \mathrm{~m} \times 250 \mathrm{~m}$ tested area processed by UCS with different $\gamma_{p}$ 's. The solid gray plots denote the peaks of the targets with $\sigma=1$; the peaks $-6 \mathrm{~dB}$ at $\gamma_{p}=0.96$ correspond to the moving target with $\sigma=0.25$.

caused by the small image pixel spacing. If the tested area is downsampled with factors of about ten in azimuth and three in range, the number of image pixels belonging to the tested area is reduced to $N_{\text {pixel }}=2500$. A similar detection of moving targets with $\mathrm{NRS} \approx 0.96$ and $\mathrm{NRS} \approx 1.04$ is then achieved with UCS.

\section{Evaluation of the Moving-Target Detection by Focusing Technique}

The moving-target detection by focusing technique can be evaluated by terms of ability of detection and processing time for the detection. The ability of detection can be based on the improvement in signal-to-clutter noise ratio (SCNR). If we define the area of detection and the area of reference by a focused moving target and a stationary point target, respectively, surrounded by low backscattering areas, the evaluation using the improvement in SCNR is introduced in [20] as follows:

$$
G_{\mathrm{SCNR}}=20 \log \left(\frac{\mu_{2}}{\mu_{1}}\right)-20 \log \left(\frac{\nu_{2}}{\nu_{1}}\right)
$$

where $\mu_{1}$ and $\mu_{2}$ are the retrieved peak intensities in the area of detection before and after focusing, respectively. Similarly, $\nu_{1}$ and $\nu_{2}$ are the peak intensities in the area of reference. Both areas of detection and reference in this simulation can be chosen relatively small. For PBP, we need only to process these small areas with different hypotheses $\gamma_{p}$ on $\gamma_{t}$. For UCS, we must process the whole SAR image and search for the peak intensities on these smaller areas.

At hypothesis $\gamma_{p}=1.04$, the energy reflected from the moving target with NRS $\approx 1.04$ is highly focused, while the energy reflected from the stationary target is dispersed. The area of detection in this test is marked by black rectangles, as shown in Fig. 2(b) and (e). The black rectangles in Fig. 2(a) and (d) delineate the area of reference containing the stationary point
TABLE II

NUMBERS OF OPERATIONS FOR DETECTION REQUIRED BY PBP AND UCS

\begin{tabular}{ll}
\hline \hline Algorithm & Number of operations \\
\hline PBP on polar & $2 \times N_{h} \times N_{a} \times N_{r} \times\left(1+\sqrt{N_{x}}\right)$ \\
PBP on slant-range & $2 \times N_{a} \times N_{r} \times\left(1+\sqrt{N_{h} \times N_{x}}\right)$ \\
UCS & $N_{h} \times N_{a} \times N_{d} \times\left[3 \times \log _{2}\left(N_{a} \times N_{d}\right)+4\right]$ \\
\hline \hline
\end{tabular}

target in the center of the SAR image. In this simulation, we can find $\mu_{1}, \mu_{2}, \epsilon_{1}$, and $\epsilon_{2}$ values by extracting information in the detection stage (solid black plots in Fig. 6 and solid gray plots in Fig. 7). The improvement in SCNR given by (39) can be visually estimated as $G_{\mathrm{SCNR}} \approx 20 \mathrm{~dB}$ for both PBP and UCS as the result of $10-\mathrm{dB}$ concentration of the moving target's energy, as well as $-10-\mathrm{dB}$ dispersion of the point target's energy. This improvement depends both on the radar system and the velocity of the moving target. With such an improvement, this technique even accommodates detecting moving objects surrounded by a considerable number of stationary targets.

The processing time for the detection is related to the computational cost. For PBP, the processing time is also affected by the subaperture length. As mentioned in Section IV-A, the focusing approach can be performed on either the polar image formation or the slant-range image formation using $\gamma_{t}$. Both proposals give advantages of processing time compared to GBP. If $N_{h}$ is the number of hypotheses, $N_{a}$ is the number of aperture positions corresponding to the integration angle handling the detection, and $N_{x}$ and $N_{r}$ are the dimensions of the tested area, the optimum numbers of aperture positions to form subapertures are given by

$$
N_{s}=\sqrt{N_{x}}
$$

for the former proposal and

$$
N_{s}=\sqrt{N_{h} \times N_{x}}
$$

for the latter one. The optimum numbers of operations for detection are summarized in Table II. It is obvious that the latter proposal even runs approximately $\sqrt{N_{h}}$ times faster than the former one.

UCS always processes a matrix of size $N_{a} \times N_{d}$ for each hypothesis $\gamma_{p}$, where $N_{d}$ is the number of range data. Since the number of range data is very large, such processing requires huge memory. In the case where the requirement of memory is fulfilled, the technique-embedded UCS runs much faster than GBP. The number of operations for detection required by UCS is also given in Table II.

\section{E. Possibility to Use the Moving-Target Detection by Focusing Technique for Estimation}

As soon as the moving target is detected, an appropriate method can be used to estimate such parameters as speed, moving direction, and dimension of the moving object. One of the NRS estimation methods, which is proposed in [35], works in an iterative way and is based on a chirp rate estimator in azimuth direction of the SAR image. The investigation in [20] 
demonstrated that the technique not only facilitates detecting moving objects but also estimating motion parameters such as NRS. Hence, a better estimate of NRS can be achieved by testing different hypotheses $\gamma_{p}$ with smaller steps close to the retrieved peak intensity. Using the same integration angle for the detection stage, a new step size for estimation $\Delta \gamma_{e}$ can be retrieved by (33) with a more strict threshold $h_{\mathrm{lim}}$. However, there are also other ways to find a step size for estimation $\Delta \gamma_{e}$, for example, the one proposed in [36] from the speed estimation's point of view. Using PBP, the extra computational cost for the NRS estimate with a smaller step size carried out by (24) is

$$
N_{h, e} \times N_{a, e} \times \frac{N_{x} \times N_{r}}{N_{s}}
$$

where $N_{h, e}$ is the number of hypotheses in the estimation stage and $N_{a, e}$ is the number of aperture positions used in the estimation stage. The estimation step can be performed on extremely small areas since small displacement caused by directional reflection has been compensated in the detection stage. Using PBP, the computational cost for this extra step is small enough to be insignificant. Estimations regarding acceleration or moving direction are possible if we test different hypotheses $\gamma_{p}$ with a smaller step and different integration angles. These aspects are not, however, presented in detail in this paper.

\section{F. Comparison Between PBP and UCS With Regard to the Moving-Target Detection by Focusing Technique}

Fig. 4(a) shows the measurement results on spatial resolution (RES), considered to be the most significant parameter for image quality measurements when evaluating quantitatively the performance of the PBP and UCS algorithms where no motion error is assumed. With regard to the detection ability, RES is related to the retrieved peak intensity, the optimum quantization step, and the image pixel spacing for detection. The measurements should also be performed at different integration angles since different azimuth focusings obtained at different integration angles result in different spatial resolutions in azimuth in UWB SAR [37]. To facilitate the comparison between PBP and UCS in terms of spatial resolution, we use the differential resolution terms (DRES) which are introduced in [37]. The references for these measurements are given by PBP spatial resolutions. The measured results are shown in Fig. 4(b). The DRES measurement results indicate that if there is no motion error, UCS can obtain approximately the same spatial resolutions in azimuth and range as PBP at small integration angles (up to $15^{\circ}$ ). These resolutions in both azimuth and range are up to $25 \%$ and $15 \%$ lower than PBP at larger integration angles, respectively. Although there is no motion error introduced, the ability of UCS to image objects distinctly and separately on a SAR image is inferior to that of PBP at large integration angles. The moving-target detection by focusing technique is associated with a large integration angle and must handle azimuth focusing for reliable detection. In such cases, PBP has advantages over UCS. Due to the approximations still existing in UCS, as well as NCS, the ability to image weak reflective targets affected by a strong reflective target nearby of these algorithms in UWB SAR is restricted. This is demonstrated by the measured results of integrated and peak sidelobe ratios given in [32]. In reality, there are always motion errors caused by any airborne SAR system, and these motion errors need to be compensated. However, there may not be a manageable motion compensation method for UCS as for timedomain algorithms [38].

As also shown in [32], the computational cost for NCS accelerates with the increase of the integration angle. This also holds for UCS. For UWB SAR processing in general and moving-target detection in UWB SAR in particular, some extra computational costs must be counted for UCS, such as elimination of higher order phase terms in the Taylor expansion (from the fourth order for NCS and the fifth order for UCS) and motion error compensation.

As shown in the tests in Section V-C, using PBP in the detection of moving target by focusing technique has the advantage of the flexible tested area, owing to PBP's local processing characteristics. In addition, the image pixel spacing can be set as desired. For UCS, the hypotheses must be tested on a larger area since its dimensions are decided by the considered number of aperture positions and the number of range data. The image pixel spacing is also limited by the required PRF and the range sampling frequency.

A comparison in terms of processing time related to the computational cost for the detection may be difficult to present in an analytical way due to the different matrix sizes to be processed. However, in our tests with simulated data, the detection-embedded UCS has no advantage in terms of processing time over PBP.

For these reasons, experiments on the real CARABAS-II data in the following sections are focused on the detectionembedded PBP. With an appropriate selection of processing parameters and an appropriate method for embedding PBP in the moving-target detection by focusing technique, this technique can be carried out efficiently both in terms of image quality for detection and processing time.

\section{First Experiment on ReAl Data}

In the first experiment on real data, we used CARABAS-II SAR data collected in the area of Simrishamn, located along the Baltic coastline in southern Sweden. The latitude and longitude of the aim point are $55.5^{\circ} \mathrm{N}$ and $14.3^{\circ} \mathrm{E}$, respectively. The parameters for the registration are given in Table I and have also been used to generate the simulated data in Sections IV and V. The imaged ground scene is shown in Fig. 8.

Using PBP, the SAR image in Fig. 8 is formed by a complete aperture of 20480 positions, equally spaced at $0.9375 \mathrm{~m}$. The dimensions are $5120 \mathrm{~m} \times 5120 \mathrm{~m}$ with 1 -m sampling in each direction. With such dimensions, an optimum subaperture size in PBP of 72 aperture positions is retrieved by (41) with $N_{h}=1$. With the available data, a selection of the tested area can be the full SAR scene $5120 \mathrm{~m} \times 5120 \mathrm{~m}$. However, due to illustration purposes, we select an area of $400 \mathrm{~m} \times 100 \mathrm{~m}$ surrounding $(1159,128)$ marked by a black rectangle in Fig. 8. In this area, moving targets can be detected, as well as imaged easily. An arbitrary integration angle $\theta=15^{\circ}$ is considered for handling the detection. Equations (37) and (38) result in $10 \mathrm{~m} \times 2.5 \mathrm{~m}$ 


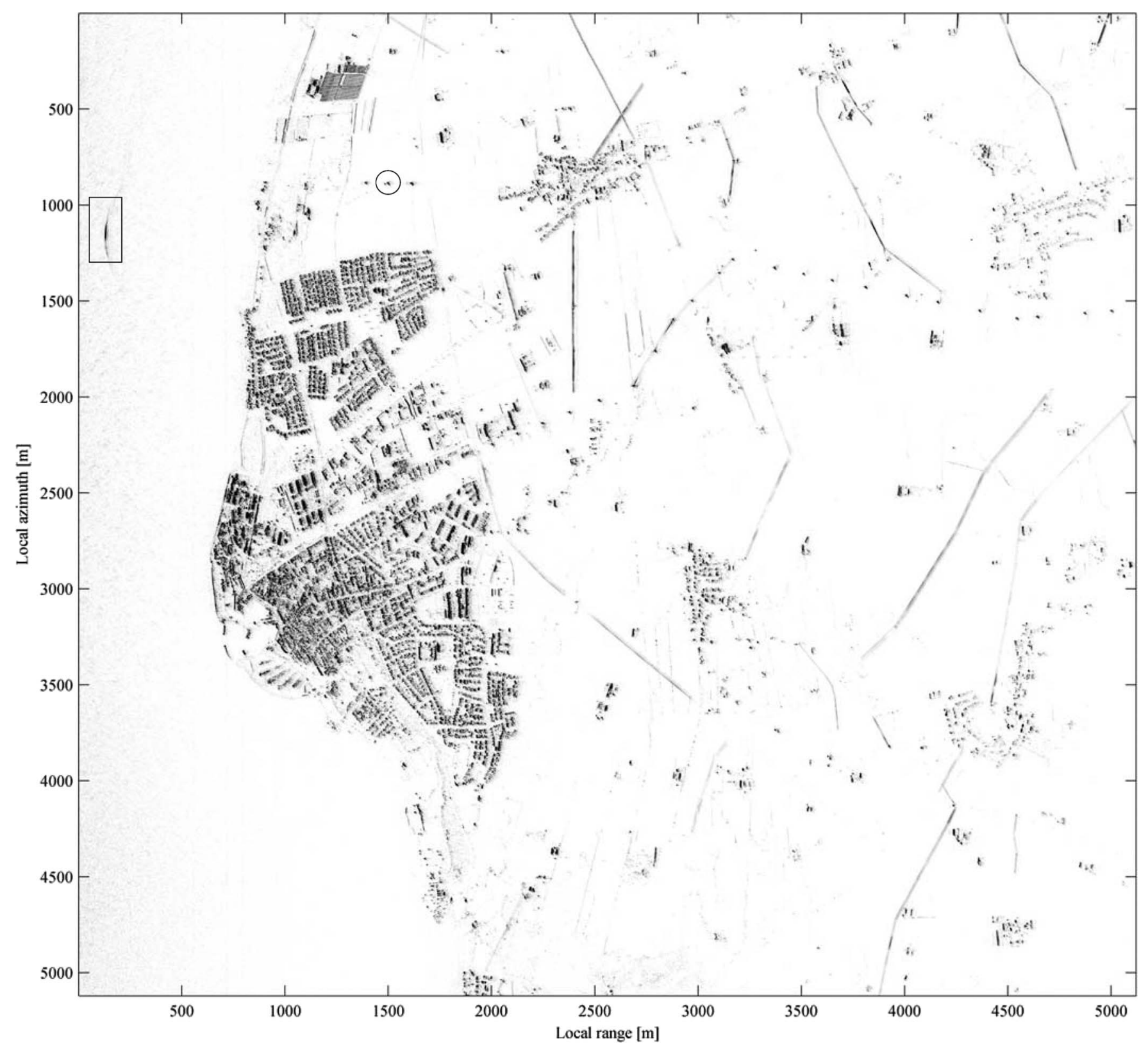

Fig. 8. Ground scene in the area of Simrishamn, located along the Baltic coastline in southern Sweden, is imaged by CARABAS-II with parameters given in Table I. The image is processed by the complete aperture which refers to an integration angle of about $110^{\circ}$ with respect to the aim point. The area surrounding a hyperbolic curve visible in a SAR image, where the presence of a moving target is predicted, is delineated by a black rectangle. A pointlike scatterer, which is marked by a black circle, is used to observe the effects of the focusing approach to the SAR image.

as the image pixel spacing. The number of image pixels belonging to the tested area is therefore $N_{\text {pixel }}=1600$. Since the selected area belongs to the territorial waters in Simrishamn, moving objects can be a boat, a ferryboat, or a ship. The speed of such vessels may be up to $50 \mathrm{~km} / \mathrm{h}$ or $14 \mathrm{~m} / \mathrm{s}$. The NRS range is set approximately from 0.9 to 1.1 based on (35) and (36). Using the parameters given in Table I, an optimum quantization step size $\Delta \gamma=7.5 \cdot 10^{-3}$ is retrieved by (33) based on the threshold $h_{\lim }=-3 \mathrm{~dB}$ and $Q=1.6$. The number of hypotheses $\gamma_{p}$ needed for detection is therefore $N_{h}=27$. Fig. 9 shows the normalized intensities of the image pixels processed with different hypotheses $\gamma_{p}$.

Measurement results on the normalized intensities indicate the presence of a moving targets with NRS $\approx 0.96$ in the tested area. The three peaks shown in Fig. 9 belong to three adjacent image pixels and should therefore be associated to one single moving target. The difference in NRS may be explained by waves in the territorial waters in Simrishamn at the time of data collection. The strong waves cause roll of the vessel, which change its velocity during the integration time. This affects severely the detection ability.

Fig. 10 shows the same imaged scene as in Fig. 8 but processed with $\gamma_{p}=0.96$. Some effects on the new formed SAR image can be seen directly. The elliptic curve in the selected area marked by the black rectangle is now focused on a small bright spot in Fig. 10. This again confirms the presence of only one vessel in the tested area. Meanwhile, pointlike scatters, e.g., the one marked by the black circle, either smear as elliptic 


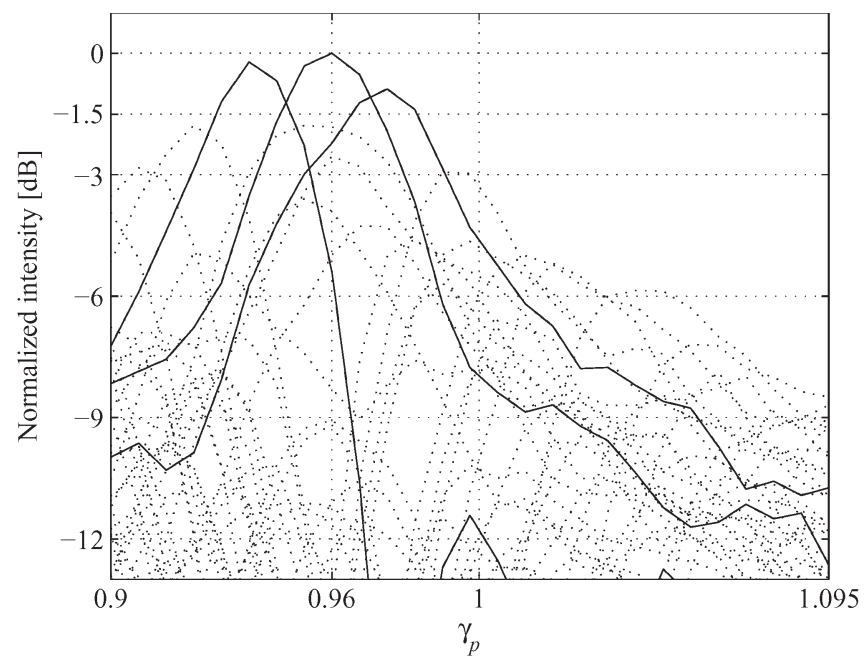

Fig. 9. Measured normalized intensities on 1600 image pixels $10 \mathrm{~m} \times 2.5$ $\mathrm{m}$, belonging to the $400 \mathrm{~m} \times 100 \mathrm{~m}$ tested area processed by PBP with different $\gamma_{p}$ 's. Optimum quantization step size for detection $\Delta \gamma=7.5 \cdot 10^{-3}$, integration angle $\theta=15^{\circ}$, and minimum range $r_{0} \approx 4700 \mathrm{~m}$. The solid black plots denote the peak values of normalized intensities.

curves or disappear since the reflected energy may drop below the clutter noise level as a result of this dispersion. The effects on the SAR image can be explained as follows. The stationary objects on the ground can now be defocused in the moving frame processed at $\gamma_{p}=0.96$ (smearing as elliptic curves). All moving objects surrounding $\gamma_{p}=0.96$ are focused to original shape. However, large stationary objects such as power lines are not affected by the focusing approach due to strong specular reflection, i.e., nonpointlike scatters.

A SAR image of the detected moving object is also shown as contour plots at levels $-3,-6,-9,-15$, and $-21 \mathrm{~dB}$ in the lower right corner of Fig. 10. The $3-\mathrm{dB}$ width of the target is estimated to be about $7 \mathrm{~m} \times 3 \mathrm{~m}$. This is a quite good result since it is close to what one would expect for an extended boat, ferryboat, or ship.

Since the moving object in this experiment is a strong scatter in a low backscattering environment, it can be visually detected at all of the proposed NRS. However, as shown in Fig. 9, the concentration of the moving target's energy at $\gamma_{p}=0.96$ is only $3 \mathrm{~dB}$ higher than that at $\gamma_{p}=1$. If the moving target was not in the territorial waters and surrounded by a number of the very strong nonpointlike scatters, the possibility to detect this moving target would be low. This specific experiment shows that applying this technique to single channel and using it directly to detect moving targets at small integration angles are suboptimal in urban areas with many strong nonpointlike scatters. In this case, a large integration angle is required to get a better improvement in SCNR for detection. Another experimental result on the data collected in the Simrishamn scene is presented in [20]. The improvement in SCNR is also about $18 \mathrm{~dB}$, however at an integration angle $\theta \approx 110^{\circ}$, i.e., the complete aperture. However, using the complete aperture is synonymous with long processing time and may violate seriously the assumptions of linear movements with constant speeds. An alternative is to combine the moving-target detection by focusing technique with multichannel techniques, e.g., DPCA and STAP, to suppress the surrounding nonpoint- like scatters [30], [39]. The improvement in SCNR obtained with the moving-target detection by focusing technique will still be preserved when combining with the multichannel techniques.

\section{SECOND EXPERIMENT ON REAL DATA}

In the second experiment on real data, we use CARABAS-II SAR data collected in a field campaign at the northern part of the island Visingsö, located in the fresh water lake Vättern in southern Sweden. The latitude and longitude of the aim point are $58.0^{\circ} \mathrm{N}$ and $14.4^{\circ} \mathrm{E}$, respectively. The parameters used in the data collection are summarized in Table III.

The imaged SAR scene processed with complete aperture is shown in Fig. 11. The dimensions of the SAR image are $9210 \times 10752$ pixels at $1 \mathrm{~m} \times 1 \mathrm{~m}$ pixel spacing. Equation (41) results in an optimum subaperture size of 104 aperture positions, equally spaced at $0.9375 \mathrm{~m}$. In this experiment, we concentrate on evaluating the gain in detection ability.

The moving object considered in this experiment is known. It is the EBBA BRAHE ferry operating in the lake Vättern. The dimensions of the ferry are $40 \mathrm{~m} \times 17 \mathrm{~m}$. At the time when the data were collected, the ferry was moving toward the island at a speed of $v_{\mathrm{tg}} \approx 10$ knots or $v_{\mathrm{tg}} \approx 5.14 \mathrm{~m} / \mathrm{s}$. The moving direction of the ferry is $\varphi \approx 180^{\circ}$ with respect to the platform velocity. Equation (34) results in an approximate true NRS $\gamma_{t}=1.04$. During this SAR data collection campaign, corner reflectors for system calibration located at different areas with low scattering background were deployed. Corner reflectors appear as point targets in a SAR image. They can therefore be seen as reference point targets and used to evaluate the gain in detection ability using (39).

\section{A. Moving-Target Detection by Focusing Experiment}

The EBBA BRAHE ferry appears in the SAR image as an elliptic curve in the area surrounding $(962,7196)$, as shown in Fig. 11. The tested area in this case is $400 \mathrm{~m} \times 100 \mathrm{~m}$ and marked by a black rectangle in Fig. 11. With the similar image pixel spacing of $10 \mathrm{~m} \times 2.5 \mathrm{~m}$, the number of image pixels is the same as the number used in the first experiment, i.e., $N_{\text {pixel }}=1600$.

The speed of the ferry is limited to $50 \mathrm{~km} / \mathrm{h}$ or $14 \mathrm{~m} / \mathrm{s}$. With given speed of the aircraft, different hypotheses $\gamma_{p}$ are tested on the selected area in an interval of 0.85 to 1.15 derived by (35) and (36). If the integration angle $\theta=15^{\circ}$ is selected for detection, an optimum quantization step size $\Delta \gamma=3 \cdot 10^{-3}$ can be estimated using (33) based on the threshold $h_{\lim }=$ $-3 \mathrm{~dB}$ and $Q=1.6$. The number of hypotheses $\gamma_{p}$ needed for detection is therefore $N_{h}=101$.

As shown in Fig. 12, the EBBA BRAHE ferry moving with an NRS $\approx 1.036$ in the tested area is detected. Since the EBBA BRAHE ferry is not strongly affected by the waves while moving in the fresh water lake Vättern, it gets more focused compared to the vessel in the first experiment. Fig. 13 shows the same imaged scene as given in Fig. 11 but processed with $\gamma_{p}=1.036$, where the moving target has the highest possibility of detection. 


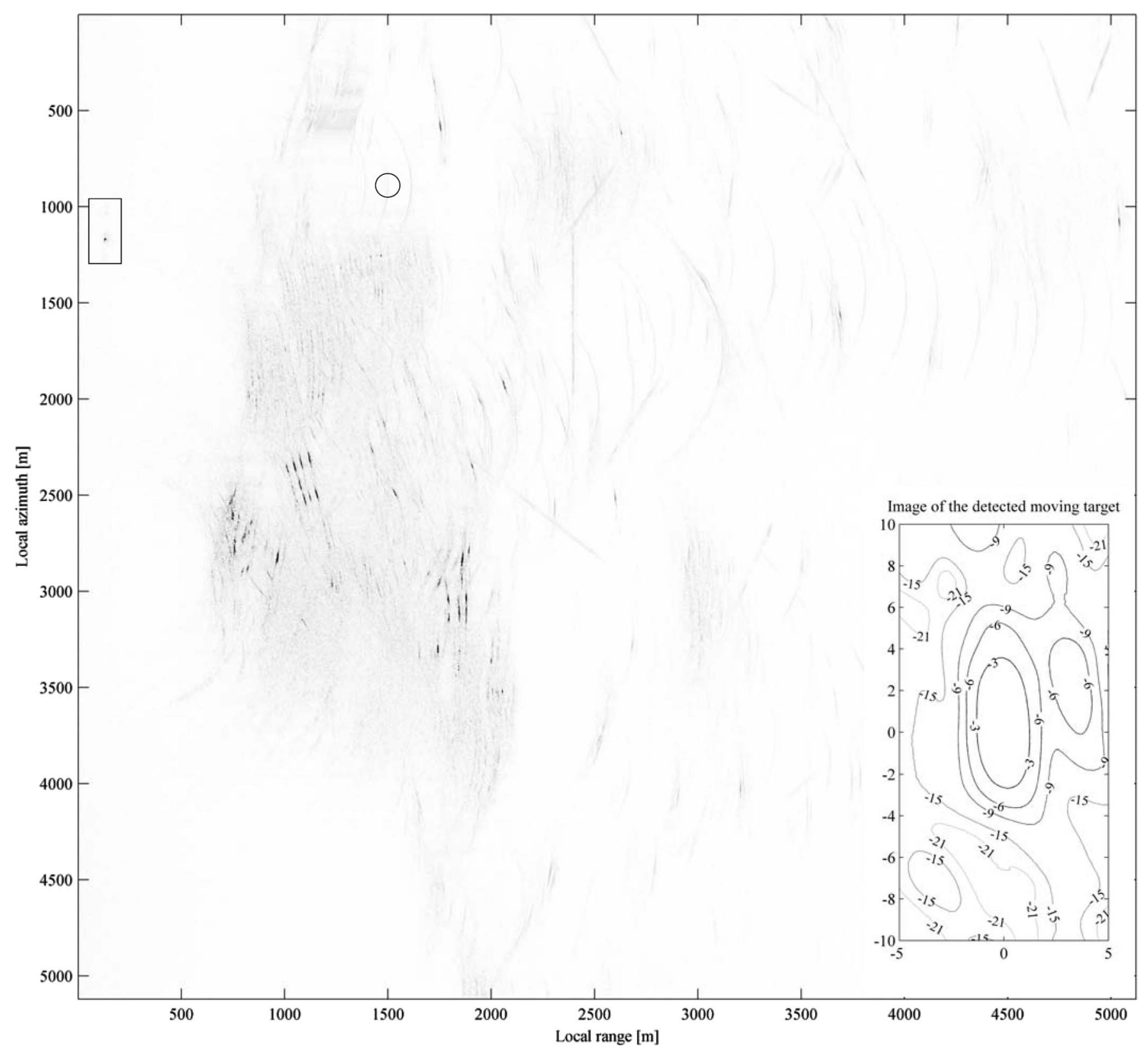

Fig. 10. Same imaged scene as in Fig. 8 but processed with $\gamma_{p}=0.96$. The image is also processed by an integration angle of about $110^{\circ}$ with respect to the aim point. The hyperbolic curve in the selected area marked by the black rectangle is now focused on a small bright spot. The pointlike scatterer marked by the black circle smears as an elliptic curve.

TABLE III

Parameters Used in the Measurement CAMPaign in Visingsö

\begin{tabular}{cc}
\hline \hline Parameter & Value \\
\hline The highest frequency processed & $82 \mathrm{MHz}$ \\
The lowest frequency processed & $22 \mathrm{MHz}$ \\
Platform speed $v_{p l}$ & $126 \mathrm{~m} / \mathrm{s}$ \\
Full aperture length & $33600 \mathrm{~m}$ \\
Full aperture positions & 35840 \\
Flight altitude & $4943 \mathrm{~m}$ \\
Minimum range to the aim point & $11250 \mathrm{~m}$ \\
PRF & $134 \mathrm{~Hz}$ \\
\hline \hline
\end{tabular}

Processing data with NRS $=1.036$ causes some effects which can be seen directly in Fig. 13. The curve found earlier in the selected area (marked by the black rectangle) is now focused on a small bright spot. Meanwhile, the corner reflector in the area of reference disappears (marked by a black circle in Fig. 11) since the energy reflected from the reflector is dispersed and drops below the clutter noise level as a result of about $-10-\mathrm{dB}$ energy loss at $\gamma_{p}=1.036$. Similar to the first experiment, large stationary objects, in this case, fences, are also not affected by defocusing.

\section{B. Evaluation of the Detection Ability}

To evaluate the detection ability using (39), an area of $50 \mathrm{~m} \times 12.5 \mathrm{~m}$ surrounding the focused EBBA BRAHE is selected to be the area of detection. As mentioned, some corner reflectors were deployed to calibrate the CARABAS-II system during this data collection campaign. One of them is located 


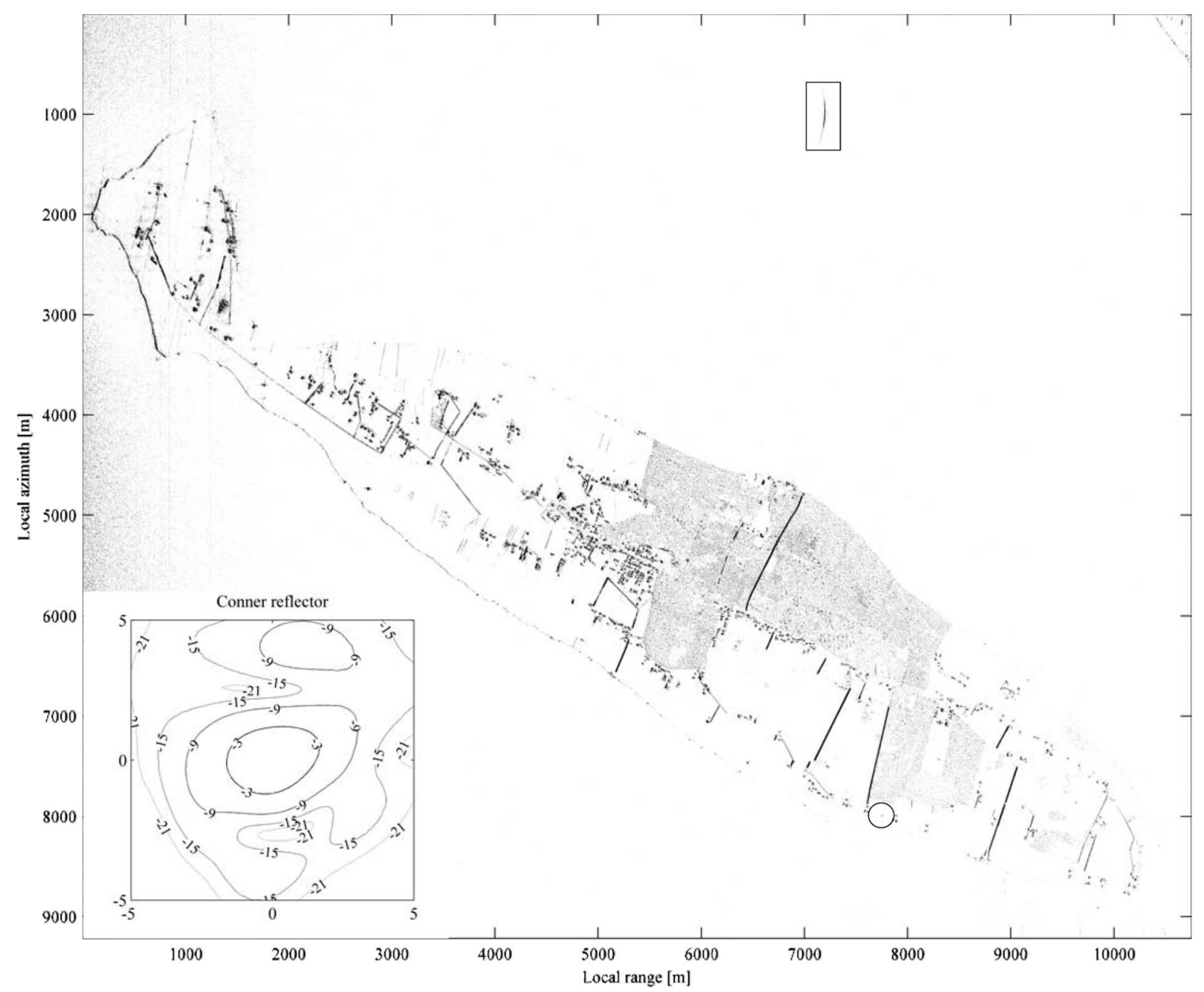

Fig. 11. Ground scene of the island Visingsö, located in the fresh water lake Vättern in southern Sweden, is imaged by CARABAS-II with the parameters given in Table III. The image is processed by the complete aperture, which refers to an integration angle of about $110^{\circ}$ with respect to the aim point. The area surrounding an elliptic curve visible in a SAR image, where the presence of a moving target is predicted, is delineated by a black rectangle. A corner reflector, which appears as a point target in the SAR image and is marked by a black circle, is used to observe the effects of the focusing approach to the SAR image, as well as evaluate the performance of the detection.

at $(7982,7751)$ in the local azimuth and range coordinates and is marked by a black circle in Fig. 11. Its contour plots at levels $-3,-6,-9,-15$, and $-21 \mathrm{~dB}$ are in the lower left corner of Fig. 11. This reflector appears in the SAR image as a point target and can be used for evaluation purposes. The area surrounding the corner reflector defines the area of reference.

As mentioned, the area can be affected by surrounding objects when the focusing approach is applied. The area of reference should thus be small enough to avoid the smearing of other objects, which may cause inexact measurements. The dimensions of the area of reference are therefore selected as $30 \mathrm{~m} \times$ $7.5 \mathrm{~m}$. The defined area of detection and area of reference are processed with different hypotheses $\gamma_{p}$. This evaluation is only based on normalized peak intensities. The normalized peak intensities measured in those areas are shown in Fig. 14 with respect to the NRS under test $\gamma_{p}$. The improvement in SCNR can be determined visually by looking at Fig. 14. In this experiment, the improvement was approximately $18 \mathrm{~dB}$ as the result of $8-\mathrm{dB}$ concentration of the moving target's energy, as well as $-10-\mathrm{dB}$ dispersion of the point target's energy. This result is similar to the SCNR obtained from the simulations in Section V-C.

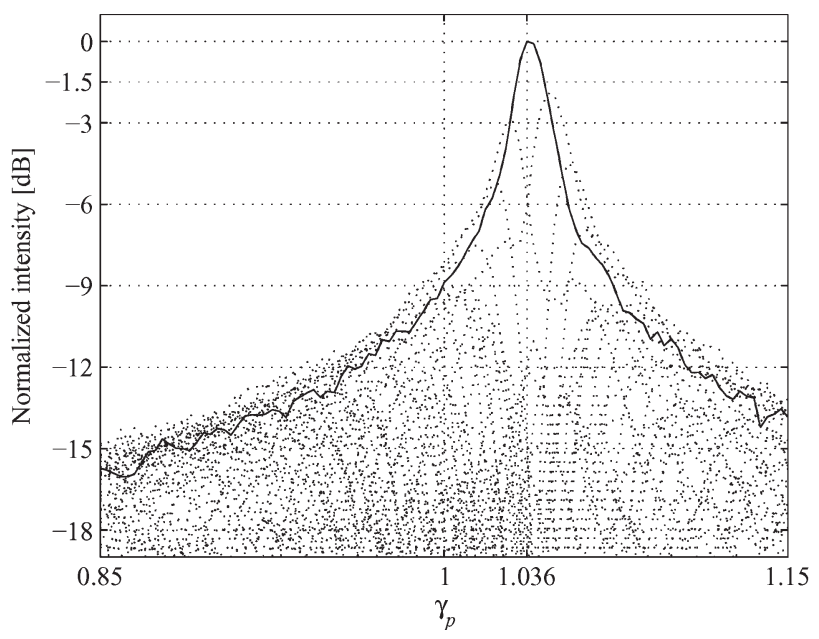

Fig. 12. Measured normalized intensities on 1600 image pixels $10 \mathrm{~m} \times 2.5 \mathrm{~m}$, belonging to the $400 \mathrm{~m} \times 100 \mathrm{~m}$ tested area processed by PBP with different $\gamma_{p}$ 's. Optimum quantization step $\Delta \gamma=3 \cdot 10^{-3}$, integration angle $\theta=15^{\circ}$, and minimum range to the object $r_{0} \approx 7600 \mathrm{~m}$. The solid black plot denotes the peak value of normalized intensities. 


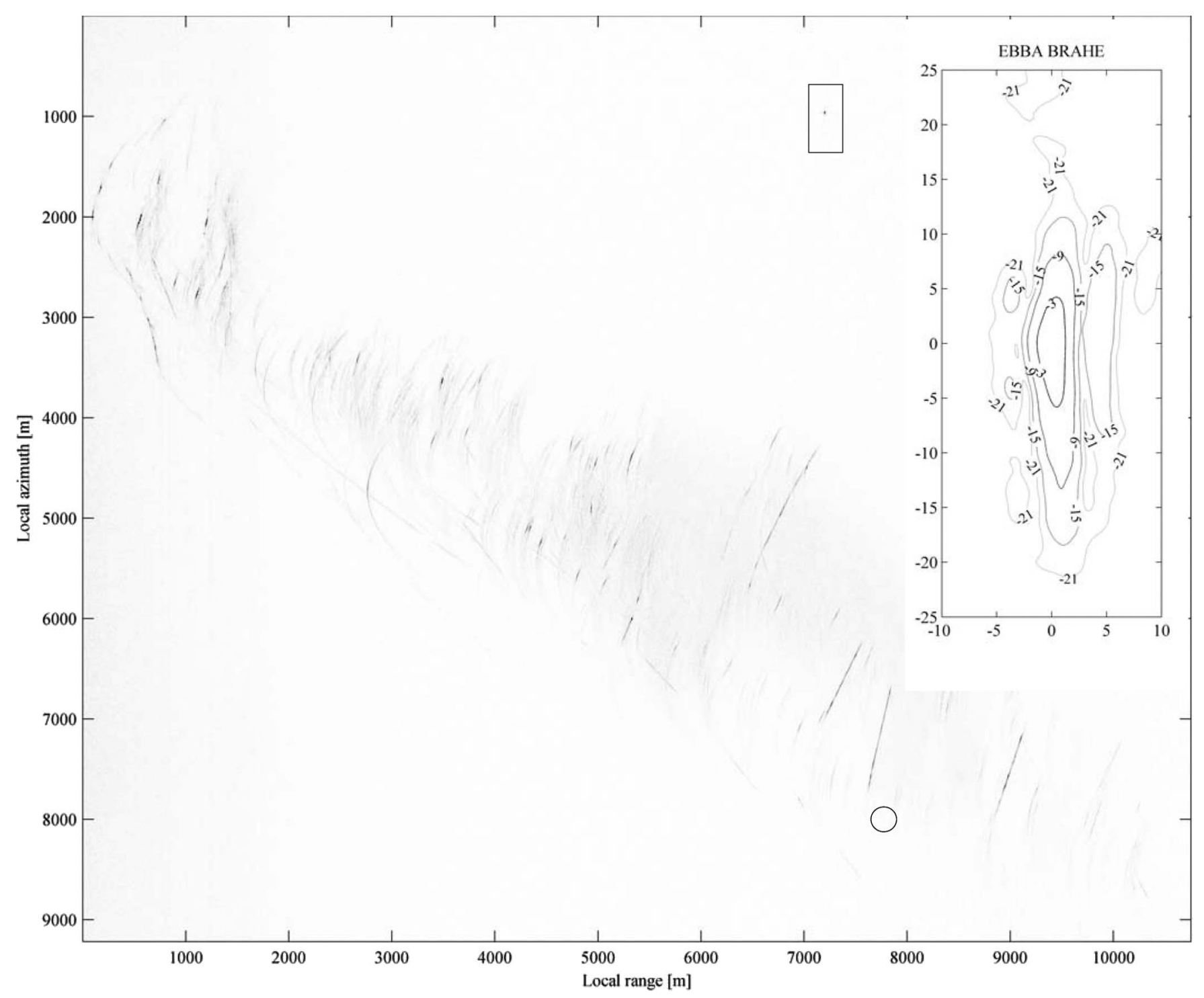

Fig. 13. Same area as in Fig. 11 but processed with $\gamma_{p}=1.036$. The image is also processed by an integration angle of about $110^{\circ}$ with respect to the aim point. Similar effects, which have been seen in Fig. 10, are again shown in this image. However, the corner reflector in the area of reference, marked by a black circle, disappears due to the energy dispersion.

\section{Moving-Target Estimation by Focusing}

NRS can be estimated by testing new hypotheses $\gamma_{p, e}$ around the NRS $=1.036$ retrieved in the detection stage. Using the same integration angle for the detection stage $\theta=15^{\circ}$, a new step size for this estimation $\Delta \gamma_{e}=6 \cdot 10^{-4}$ is retrieved by (33) with a more strict threshold $h_{\lim }=-0.1 \mathrm{~dB}$ corresponding to $Q=0.7$. The new hypotheses $\gamma_{p, e}$ are tested on the interval $[\mathrm{NRS}-\Delta \gamma, \mathrm{NRS}+\Delta \gamma]$, i.e., $[1.033,1.039]$. The number of hypotheses $\gamma_{p, e}$ needed for estimation is therefore $N_{h, e}=11$. The image pixel spacing for estimation can be reduced, for example, to $0.5 \mathrm{~m} \times 0.5 \mathrm{~m}$. The new hypotheses $\gamma_{p, e}$ are tested on a smaller area of $10 \mathrm{~m} \times 2.5 \mathrm{~m}$, which is called the area of estimation. The number of image pixels is $N_{\text {pixel }}=100$. An investigation of the normalized peak intensities around $\mathrm{NRS}=1.036$ is shown in Fig. 15.

A more accurate NRS $=1.0372$ is retrieved in this estimation stage. Hence, the moving-target detection by focusing technique, when applied with a smaller step size than the optimum for detection, can be seen as an NRS estimation method. However, if we perform the NRS estimation method with different integration angles, other motion parameters may be retrieved. In this experiment, we perform the NRS estimation method with another integration angle, i.e., $\theta=30^{\circ}$, but use the same step size $\Delta \gamma_{e}=6 \cdot 10^{-4}$. The retrieved peak intensities corresponding to different $\gamma_{p, e}$ 's is also shown in Fig. 15. The maximum value of the peak intensities is reached at $\gamma_{p, e}=$ 1.0378. The new estimated NRS $=1.0378$ is different from the old estimated value of 1.0372 . In our other experiments where we estimated the NRS with the complete aperture, NRS $=1.04$ was retrieved. The differences may be caused in part by the acceleration or the directional change of the moving object during the time interval of data collection. The movement of the ferry caused by sea waves and wind gives also rise to an imprecise estimation of NRS. The effects of such factors to NRS estimation are proportional to the increase of the integration angle. The different estimated NRSs by different integration angles $\theta$ open also possibilities to estimate the speed, the acceleration, and, therefore, the moving direction of 


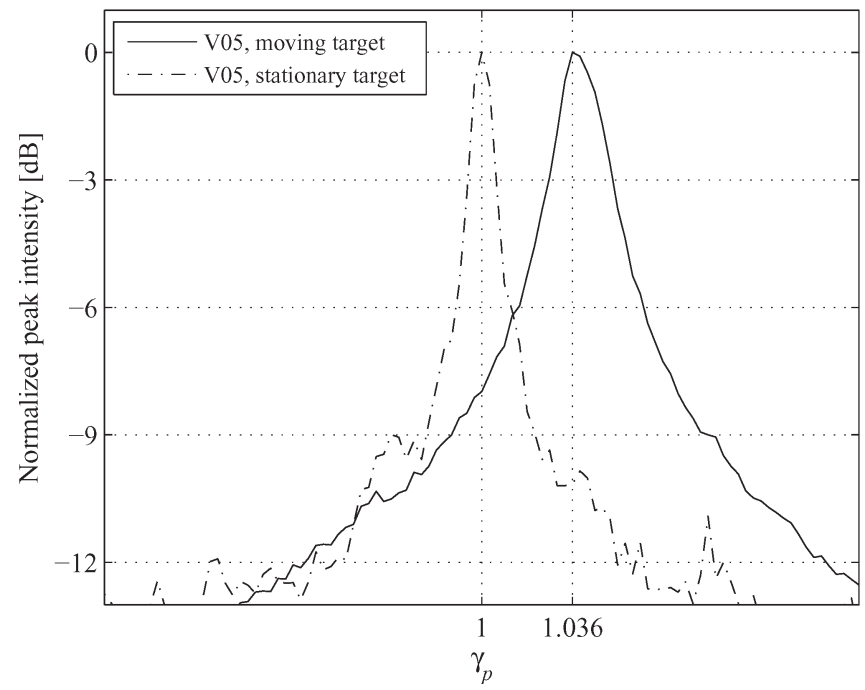

Fig. 14. Measured normalized peak intensities on the area of detection $50 \mathrm{~m} \times$ $12.5 \mathrm{~m}$ and the area of reference $30 \mathrm{~m} \times 7.5 \mathrm{~m}$ processed by PBP with different $\gamma_{p}$ 's. Optimum quantization step $\Delta \gamma=3 \cdot 10^{-3}$, integration angle $\theta=15^{\circ}$, and minimum range to the object $r_{0} \approx 7600 \mathrm{~m}$.

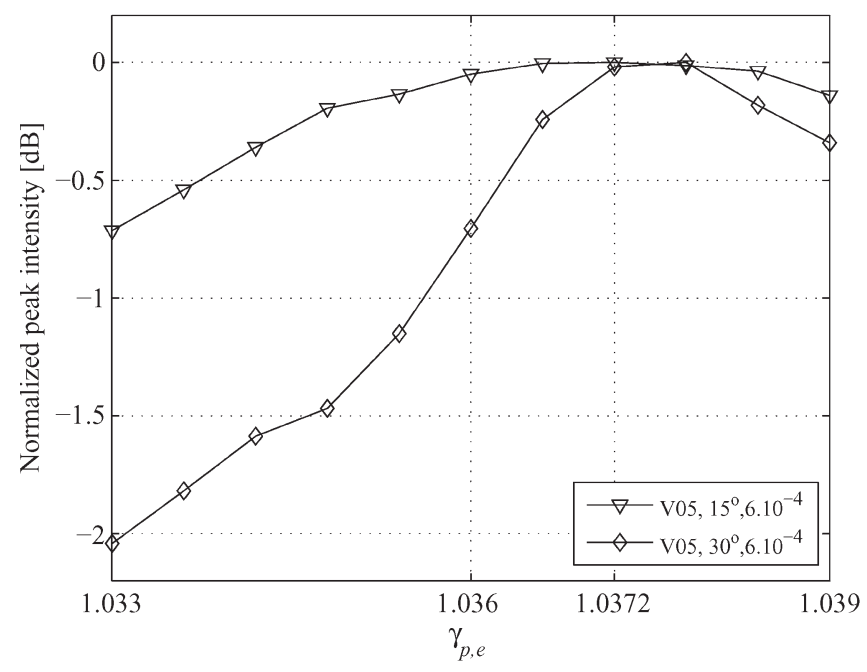

Fig. 15. Measured normalized peak intensities on the area of estimation $10 \mathrm{~m} \times 2.5 \mathrm{~m}$ processed by PBP with different $\gamma_{p}$ 's. Optimum quantization step $\Delta \gamma=6 \cdot 10^{-4}$, integration angle $\theta=15^{\circ}$ and $\theta=30^{\circ}$, and minimum range $r_{0} \approx 7600 \mathrm{~m}$.

the moving object by nonlinear tracking filters, i.e., Kalman filters [40]. For the multichannel cases, such estimations can be simplified and accurate with the estimated NRS. However, these topics will not be presented in this paper.

The small figure in the upper right corner in Fig. 13 shows the contour plot of the EBBA BRAHE ferry after being focused with the estimated NRS $=1.0378$. Contour plots at $-3,-9$, -15 , and $-21 \mathrm{~dB}$ are considered. A $3-\mathrm{dB}$ reduction in the peak signal power corresponds to the smallest curve in the center of the contour plot. The dimensions of the $-3-\mathrm{dB}$ contour are approximately $10 \mathrm{~m} \times 3 \mathrm{~m}$. This should not be considered as the dimensions of the ferry but rather as the strongest reflector on the starboard side. In this case, the ferry has its large board side against the radar with a triangular shape with the wheelhouse on the top. This triangle probably causes the reflection with a maximum at the wheelhouse. Looking at the total reflection, it has an extension of 30-40 m, which is in agreement of the length of the ferry board. Apart from this long reflector is another one that is probably related to the second side of ferry, i.e., the port side of the car deck.

\section{CONCLUSION}

Moving-target detection by focusing is a promising technique when UWB SAR systems are used, particularly at low frequencies. Both time- and frequency-domain algorithms can be integrated into this technique, i.e., as the basic prerequisite for the focusing approach. The moving-target detection by focusing technique does not require multichannel data. However, applying this technique to single channel is suboptimal. A combination of this technique with multichannel techniques, e.g., DPCA and STAP to suppress clutter, will provide even better detection and estimation results. An important advantage of using this technique is that the ability of detection increases significantly even when a moving target may be surrounded by a considerable number of stationary targets or clutter. In the experiment on the simulated data, the improvement in SCNR is up to $20 \mathrm{~dB}$ at an integration angle of $\theta=15^{\circ}$. The second experimental results on the CARABAS-II data show a similar improvement in SCNR (about $18 \mathrm{~dB}$ ) using the same integration angle. This gain will be at least preserved when combining with multichannel techniques.

The selection of integration angle for detection is critical. Wide integration angle allows better azimuth focusing and, therefore, higher possibility of the moving-target detection. However, this is synonymous with long processing time and may seriously violate assumptions such as linear movements.

A fast time-domain algorithm PBP integrated into the moving-target detection by focusing technique can easily be implemented on small areas with a manageable motion compensation. This may be a challenge for frequency-domain algorithms. The investigations in this paper have also shown that the number of operations needed for the detection also depends on the method for embedding the PBP. In the experiments where we concentrate on illustration and evaluation, the technique works well in the sea environments. However, to get a better performance and shorter processing time in reality, processing small areas and parallel processing are advised. The technique also shows the ability to estimate NRS and to indicate the existence of acceleration, as well as changing direction. Other motion parameters, such as speed, acceleration, and, therefore, the moving direction are also possible to be estimated.

\section{ACKNOWLEDGMENT}

The authors would like to thank the KK Foundation for making this research project possible.

\section{REFERENCES}

[1] A. Gustavsson, L. M. H. Ulander, B. H. Flood, P.-O. Frölind, H. Hellsten, T. Jonsson, B. Larsson, and G. Stenstrom, "Development and operation of an airborne VHF SAR system-Lessons learned," in Proc. IEEE IGARSS, Seattle, WA, Jul. 1998, vol. 1, pp. 458-462.

[2] L. M. H. Ulander and H. Hellsten, "Low-frequency ultrawideband array-antenna SAR for stationary and moving target imaging," in Proc. SPIE Radar Sensor Technology IV, Orlando, FL, Apr. 1999, vol. 3704, pp. $149-158$. 
[3] D. R. Sheen, C. M. Strawitch, and T. B. Lewis, "UHF wideband SAR design and preliminary results," in Proc. IEEE IGARSS, Pasadena, CA, Aug. 1994, vol. 1, pp. 289-291.

[4] L. Carin, N. Geng, M. McClure, J. Sichina, and L. Nguyen, "Ultra-wideband synthetic-aperture radar for mine-field detection," IEEE Antennas Propag. Mag., vol. 41, no. 1, pp. 18-33, Feb. 1999.

[5] A. R. Brenner and J. H. G. Ender, "Demonstration of advanced reconnaissance techniques with the airborne SAR/GMTI sensor PAMIR," Proc. Inst. Elect. Eng.-Radar Sonar Navig., vol. 153, no. 2, pp. 152-162, Apr. 2006.

[6] L. M. H. Ulander, M. Lundberg, W. Pierson, and A. Gustavsson, "Change detection for low-frequency SAR ground surveillance," Proc. Inst. Elect. Eng.-Radar Sonar Navig., vol. 152, no. 6, pp. 413-420, Dec. 2005.

[7] J. K. Jao, "Theory of synthetic aperture radar imaging of a moving target," IEEE Trans. Geosci. Remote Sens., vol. 39, no. 9, pp. 1984-1992, Sep. 2001.

[8] R. Linnehan, L. Perlovsky, I. C. Mutz, M. Rangaswamy, and J. Schindler, "Detecting multiple slow-moving targets in SAR images," in Proc. IEEE Sensor Array Multichannel Signal Process. Workshop, Barcelona, Spain, Jul. 2004, pp. 643-647.

[9] M. I. Skolnik, Radar Handbook, 2nd ed. New York: McGraw-Hill, 1990, ch. 16 , pp. $8-17$.

[10] L. E. Brennan, J. D. Mallett, and I. S. Reed, "Adaptive arrays in airborne MTI radar," IEEE Trans. Antennas Propag., vol. AP-24, no. 5, pp. 607615, Sep. 1976.

[11] J. H. G. Ender, "Space-time processing for multichannel synthetic aperture radar," Proc. Inst. Elect. Eng.-Electron. Commun. Eng. J., vol. 11, no. 1, pp. 29-38, Feb. 1999.

[12] M. Jahangir and C. P. Moate, "Utilising signal absence in SAR imagery for moving target detection," in Proc. IET Forum Waveform Diversity Des. Commun. Radar Sonar, London, U.K., Nov. 2006, pp. 41-46.

[13] J. R. Fienup, "Detecting moving targets in SAR imagery by focusing," IEEE Trans. Aerosp. Electron. Syst., vol. 37, no. 3, pp. 749-809, Jul. 2001.

[14] M. Rüegg, E. Meier, and D. Nüesch, "Capabilities of dual-frequency millimeter wave SAR with monopulse processing for ground moving target indication," IEEE Trans. Geosci. Remote Sens., vol. 45, no. 3, pp. 539553, Mar. 2007.

[15] C. Liu and C. H. Gierull, "A new application for PolSAR imagery in the field of moving target indication/ship detection," IEEE Trans. Geosci. Remote Sens., vol. 45, no. 2, pp. 3426-3436, Nov. 2007.

[16] M. I. Pettersson, "Detection of moving targets in wideband SAR," IEEE Trans. Aerosp. Electron. Syst., vol. 40, no. 3, pp. 780-796, Jul. 2004.

[17] M. I. Pettersson, "Optimum relative speed discretisation for detection of moving objects in wide band SAR," IET Radar Sonar Navig., vol. 1, no. 3 , pp. 213-220, 2007.

[18] S. Barbarossa, "Detection and imaging of moving objects with synthetic aperture radar. 1. Optimal detection and parameter estimation theory," Proc. Inst. Elect. Eng._Radar Signal Process., vol. 139, no. 1, pp. 7988, Feb. 1992.

[19] O. Seger, M. Herberthson, and H. Hellsten, "Real time SAR processing of low frequency ultra wide band radar data," in Proc. EUSAR, Friedrichshafen, Germany, May 1998, pp. 489-492.

[20] V. T. Vu, T. K. Sjögren, M. I. Pettersson, H.-J. Zepernick, and A. Gustavsson, "Experimental results on moving targets detection by focusing in UWB low frequency SAR," in Proc. IET RADAR, Edinburgh, U.K., Oct. 2007, pp. 562-566.

[21] V. T. Vu, T. K. Sjögren, and M. I. Pettersson, "Moving target detection by focusing for frequency domain algorithms in UWB low frequency SAR," in Proc. IEEE IGARSS, Boston, MA, Jul. 2008, pp. 161-164.

[22] L. E. Andersson, "On the determination of a function from spherical averages," SIAM J. Math. Anal., vol. 19, no. 1, pp. 214-232, Jan. 1988

[23] A. F. Yegulalp, "Fast backprojection algorithm for synthetic aperture radar," in Proc. IEEE RadarCon, Waltham, MA, Apr. 1999, pp. 60-65.

[24] M. I. Pettersson, "Focusing of moving targets in an ultra wide band SAR GMTI system," in Proc. EUSAR, Munich, Germany, May 2000, pp. $837-840$.

[25] L. M. H. Ulander, H. Hellsten, and G. Stenstrom, "Synthetic-aperture radar processing using fast factorized back-projection," IEEE Trans. Aerosp. Electron. Syst., vol. 39, no. 3, pp. 760-776, Jul. 2003.

[26] P.-O. Frölind and L. M. H. Ulander, "Evaluation of angular interpolation kernels in fast back-projection SAR processing," Proc. Inst. Elect. Eng._Radar Sonar Navig., vol. 153, no. 3, pp. 243-249, Jun. 2006.

[27] C. Cafforio, C. Prati, and F. Rocca, "SAR data focusing using seismic migration techniques," IEEE Trans. Aerosp. Electron. Syst., vol. 27, no. 2, pp. 194-207, Mar. 1991.
[28] V. T. Vu, T. K. Sjögren, and M. I. Pettersson, "Ultra-wideband chirp scaling," IEEE Geosci. Remote Sens. Lett., vol. 7, no. 2, pp. 282-286, Apr. 2010.

[29] V. T. Vu, T. K. Sjögren, and M. I. Pettersson, "Fast detection of moving targets by focusing in ultra-wideband SAR," in Proc. IEEE RadarCon, Pasadena, CA, May 2009, pp. 1-5.

[30] V. T. Vu, T. K. Sjögren, and M. I. Pettersson, "Fast detection of moving targets by focusing in multi-channel ultra-wideband SAR," in Proc. EuRAD, Rome, Italy, Sep. 2009, pp. 218-221.

[31] R. K. Raney, H. Runge, R. Bamler, I. G. Cumming, and F. H. Wong, "Precision SAR processing using chirp scaling," IEEE Trans. Geosci. Remote Sens., vol. 32, no. 4, pp. 786-799, Jul. 1994.

[32] V. T. Vu, T. K. Sjögren, and M. I. Pettersson, "A comparison between fast factorized backprojection and frequency-domain algorithms in UWB low frequency SAR," in Proc. IEEE IGARSS, Boston, MA, Jul. 2008, pp. 1293-1296.

[33] G. W. Davidson, I. G. Cumming, and M. R. Ito, "A chirp scaling approach for processing squint mode SAR data," IEEE Trans. Aerosp. Electron. Syst., vol. 32, no. 1, pp. 121-133, Jan. 1996.

[34] L. M. H. Ulander and H. Hellsten, "A new formula for SAR spatial resolution," AEÜ Int. J. Electron. Commun., vol. 50, no. 2, pp. 117-121, 1996.

[35] T. K. Sjögren, V. T. Vu, M. I. Pettersson, H.-J. Zepernick, and A. Gustavsson, "Speed estimation experiments for ground moving targets in UWB SAR," in Proc. IET RADAR, Edinburgh, U.K., Oct. 2007, pp. 519-523.

[36] V. T. Vu, T. K. Sjögren, M. I. Pettersson, and P. A. C. Marques, "Application of moving target detection by focusing technique in civil traffic monitoring," in Proc. IEEE IGARSS, Honolulu, HI, 2010, to be published.

[37] V. T. Vu, T. K. Sjögren, M. I. Pettersson, and A. Gustavsson, "Definition on SAR image quality measurements for UWB SAR," in Proc. SPIE Image Signal Process. Remote Sens. XIV, Cardiff, U.K., Sep. 2008, vol. 7109, pp. 71091A-1-71091A-9.

[38] O. Frey, C. Magnard, M. Rüegg, and E. Meier, "Focusing of airborne synthetic aperture radar data from highly non-linear flight tracks," IEEE Trans. Geosci. Remote Sens., vol. 47, no. 6, pp. 1844-1858, Jun. 2009.

[39] V. T. Vu, T. K. Sjögren, and M. I. Pettersson, "Integrating spacetime processing into time-domain backprojection process to detect and image moving object," in Proc. IEEE IGARSS, Honolulu, HI, 2010, to be published.

[40] B. Ristic, S. Arulampalam, and N. Gordon, Beyond the Kalman Filter: Particle Filters for Tacking Application. London, U.K.: Artech House, 2004, ch. 5, pp. 85-102.

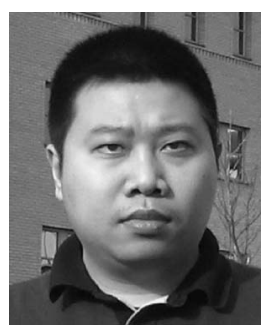

Viet Thuy Vu (S'06) was born in Hanoi, Vietnam, in 1977. He received the Diploma degree in electronics from the Hanoi University of Technology, Hanoi, in 1999, the M.Sc. degree in communication engineering from the University of Duisburg-Essen, Duisburg, Germany, in 2006, and the Licentiate degree in applied signal processing from the Blekinge Institute of Technology (BTH), Ronneby, Sweden, in 2009 , where he is currently working toward the Ph.D. degree in radar signal processing.

Since 2006, he has been with the Department of Electrical Engineering, BTH. His research interests are mono- and bistatic UWB SAR signal processing and applications of UWB SAR in moving-target detection and estimation.

Mr. Vu was the recipient of the Student Paper Prize at the IET International Conference on Radar Systems in 2007.

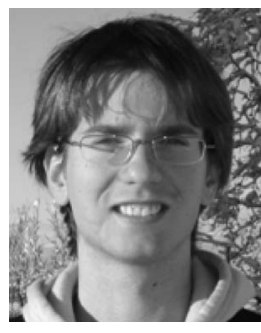

Thomas K. Sjögren (S'06) was born in Sundsvall, Sweden, in 1982. He received the M.Sc. degree in space engineering from the Luleå University of Technology, Luleå, Sweden, in 2006 and the Licentiate degree in applied signal processing from the Blekinge Institute of Technology (BTH), Ronneby, Sweden, in 2010, where he is currently working toward the $\mathrm{Ph} . \mathrm{D}$. degree in radar signal processing.

Since 2006, he has been with the Department of Electrical Engineering, BTH. His research interests include detection of moving targets and estimation of target parameters in SAR, particularly wideband SAR. 


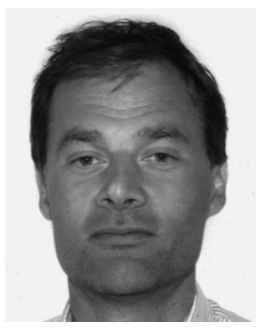

Mats I. Pettersson (M'05) received the M.Sc. degree in engineering physics, the Licentiate degree in radio and space science, and the Ph.D. degree in signal processing from the Chalmers University of Technology, Gothenburg, Sweden, in 1993, 1995, and 2000, respectively.

From 1993 to 1995 , he was with Chalmers as a Ph.D. student working with radar scatterometer measurements, modeling electromagnetic scattering, and synthetic aperture radar (SAR) image processing. For nearly two years, in 1996-1997, he was a Patent Project Manager with Ericsson Mobile Communication, Lund, Sweden. From 1998 to 2007, he was with the Division of Sensor Technology, Swedish Defence Research Agency, Linköping, Sweden, where he was a Research Manager and a Researcher, mainly focusing on ultrawideband low-frequency SAR systems. Since 2005, he has been with the Department of Electrical Engineering, Blekinge Institute of Technology, Ronneby, Sweden. This work is related to radar and sonar signal processing. His main interests are SAR processing, space time adaptive processing, and underwater sparse passive arrays.

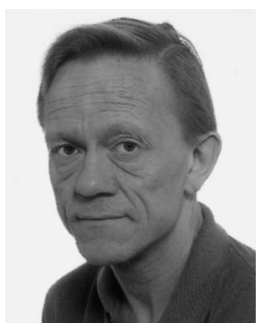

Anders Gustavsson received the M.Sc. degree in applied physics and electrical engineering from Linköping University, Linköping, Sweden, in 1982.

In 1982, he joined the Swedish Defence Research Agency, Linköping, to work with the implementation of a computer-based SAR processor for microwave systems. He has been involved in the development of airborne low-frequency SAR systems since 1990, where his current work mainly concerns signal processing issues. He has participated in a number of field campaigns with airborne and spaceborne SAR sensors, focusing primarily on forestry and foliage penetration problems.

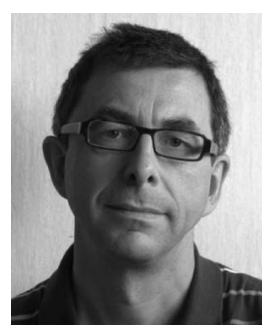

Lars M. H. Ulander (S'86-M'90-SM'04) received the M.Sc. degree in engineering physics and the $\mathrm{Ph} . \mathrm{D}$. degree in electrical and computer engineering from the Chalmers University of Technology, Gothenburg, Sweden, in 1985 and 1991, respectively.

Since 1995, he has been with the Swedish Defence Research Agency, Linköping, Sweden, where he is currently the Director of Research in radar signal processing. He is also an Adjunct Professor in radar remote sensing with the Chalmers University of Technology. His research areas are synthetic aperture radar, electromagnetic scattering models, and remote sensing applications. He is the author or coauthor of over 250 professional publications, of which more than 40 are in peer-reviewed scientific journals. He is the holder of five patents and is also a member of the Remote Sensing Committee, Swedish National Space Board. 